\title{
Orbital free ab initio molecular dynamics simulation study of some static and dynamic properties of liquid noble metals
}

\author{
G.M. Bhuiyan ${ }^{1}$, L.E. Gonzále $z^{2}$, D.J. Gonzále $z^{-2}$ \\ ${ }^{1}$ Department of Theoretical Physics, University of Dhaka, Dhaka-1000, Bangladesh \\ 2 Departamento de Física Teórica, Universidad de Valladolid, Valladolid, Spain \\ Received June 19, 2012, in final form July 31, 2012
}

Several static and dynamic properties of liquid $\mathrm{Cu}, \mathrm{Ag}$ and $\mathrm{Au}$ at thermodynamic states near their respective melting points, have been evaluated by means of the orbital free ab-initio molecular dynamics simulation method. The calculated static structure shows good agreement with the available $\mathrm{X}$-ray and neutron diffraction data. As for the dynamic properties, the calculated dynamic structure factors point to the existence of collective density excitations along with a positive dispersion for I-Cu and I-Ag. Several transport coefficients have been obtained which show a reasonable agreement with the available experimental data.

Key words: liquid noble metals, orbital free density functional theory, molecular dynamics simulations, static structure, dynamic properties, transport coefficients

PACS: $61.25 . \mathrm{Mv}, 64.70 . F x, 71.15 . P d$

\section{Introduction}

The $d$-electrons in the $d$-band metals are not so free as to justify a nearly free electron (NFE) approach but, on the other hand, they are not so tightly bound as to be described by the tight binding method (TBM) or core electron theory. Indeed, the study of $d$-band metals poses difficult theoretical challenges although some progress has been made towards their understanding, both in the solid and liquid phases [1 -11].

From a theoretical point of view, accurate first principles electronic structure calculations of $d$-band metals have been performed using the techniques such as the linearized augmented plane wave or the linearized muffin-tin orbital (LMTO) methods [12]. Although it is possible to accurately calculate the interionic forces within these schemes [13, 14], still the computational demand of such calculations has so far prevented its use within the context of Molecular Dynamics (MD) simulations. As a consequence, most realistic structural models for $d$-electron systems have been constructed by means of empirical or semiempirical interatomic potentials [15-21].

In the particular case of the noble metals, the $d$-bands are completely filled but the $s p$ - $d$ hybridization is still there [4, 22]. This $s p-d$ hybridization effect can be accounted for by either changing the $s, p, d$ band occupancy number (in the case of ab-initio pseudopotential theory) or by using an effective valence $Z$ [23]. In this respect, it has already been found from the density functional based generalized pseudopotential theory that the effective $s p$-electron valence lies [5-7] within the range 1.1 to 1.7 , where this non-integral number is mostly due to $s p$ - $d$ hybridization effects [5-7, 22, 23].

The structure of the liquid noble metals has been studied at several temperatures by Waseda [24] using X-ray (XR) diffraction methods. Neutron diffraction has been also used in the case of $\mathrm{Cu}$ at two temperatures [25] and Ag near melting [26]. As concerns their thermophysical properties, the situation is different in the case of $\mathrm{l}-\mathrm{Cu}$ and $\mathrm{l}-\mathrm{Ag}$ on the one hand, and l-Au on the other hand. Two recent compilations of thermophysical properties of liquid metals, due to Blairs [27] and Singh et al. [28], the latter including several temperatures, analyze the previous experimental measurements of the adiabatic sound 
velocity $\left(c_{\mathrm{S}}\right)$, density $(\rho)$, and specific heat at a constant pressure $\left(C_{\mathrm{P}}\right)$ of the systems, and therefrom deduce several other magnitudes, such as the isothermal compressibility $\left(\kappa_{\mathrm{T}}\right)$ or the ratio of specific heats at a constant pressure and at a constant volume $(\gamma)$. Now, in the cases of $\mathrm{l}-\mathrm{Cu}$ and $\mathrm{l}-\mathrm{Ag}$, several experimental measurements were available, so an assessment was performed and recommended values were given by the authors [27, 28]. On the contrary, only a single experiment is available to determine the sound velocity of l-Au, within a wider study of the Au-Co alloy [29]. Therefore, one should consider that the uncertainty in the thermophysical data of l-Au is larger that for l-Cu and l-Ag. Other transport properties of the liquid noble metals, such as self-diffusion coefficient $(D)$, or shear viscosity $(\eta)$ are readily available [30, 31]. In particular, the self-diffusion coefficients of l-Cu over a wide temperature range, have recently been determined by means of quasielastic neutron scattering measurements [32]. More specifically, the experimental data were used to calculate the self intermediate scattering functions, $F_{\mathrm{s}}(q, t)$, at several $q$-values, and the associated self-diffusion coefficients were evaluated from their decay rate at small wavevectors.

Most theoretical studies on the liquid noble metals have focused on the static structural properties and thermodynamic properties, usually characterizing the liquid system by effective interatomic potentials constructed either empirically by fitting to some experimental data or derived from some approximate theoretical model. Therefrom, the liquid structure is determined by resorting to either liquid state theories [33] or to classical molecular dynamics (CMD) simulations.

Holender et al. [34] have used the embedded atom model (EAM) to obtain some effective interatomic potentials which were later on used in CMD simulations aimed at evaluating the static structure of liquid noble metals near melting. Bogicevic et al. [35] used the effective medium theory to obtain a many-body potential which, combined with CMD simulations, provided information on the static properties and the self-diffusion coefficient of l-Au at different temperatures. Their calculated pair distribution function, $g(r)$, near melting has the main peak which is somewhat lower than experiment and the subsequent oscillations are slightly out of phase.

Alemany et al. [36-39] used both the EAM and TBM to derive many-body potentials which were used in CMD simulations so as to obtain information on various static and dynamic properties of $1-\mathrm{Cu}, \mathrm{l}-\mathrm{Ag}$ and l-Au. Their calculated static structure factors, $S(q)$, showed a good agreement with experiment except for a somewhat smaller height of the main peak. They also obtained reasonable estimates for the self-diffusion coefficients excepting l-Cu which was clearly underestimated. A similar approach was used by Han et al. [40] to evaluate the self-diffusion and shear viscosity coefficients in liquid and undercooled $\mathrm{Cu}$. We also note that other workers [21, 41, 42] have resorted to integral equation-type liquid state theories which, combined with semiempirical interatomic potentials, have lead to reasonable estimates for several static and thermodynamic properties of a range of $3 d, 4 d$ and $5 d$ liquid transition metals.

In principle, an accurate approach to the study of the static and dynamic properties of the liquid noble metals, would be provided by ab-initio molecular dynamics (AIMD) simulation methods, which have become widespread in the last twenty years or so. Most AIMD methods are based on the density functional theory (DFT) [43, 44] which permits to calculate the ground state electronic energy of a collection of atoms, for given nuclear positions, as well as yields the forces on the nuclei via the Hellmann-Feynman theorem. It enables one to perform MD simulations in which the nuclear positions evolve according to classical mechanics whereas the electronic subsystem follows adiabatically. The Kohn-Sham (KS) orbital representation of the DFT (KS-AIMD method) has been the usual approach when performing AIMD simulations although it is acknowledged that this approach imposes heavy computational demands which limit the size of the systems as well as the simulation times. These limitations are enhanced in the case of $d$-electron systems such as the noble and transition metals because a large number of electronic orbitals are needed. Nevertheless, and despite the above shortcomings, a few AIMD studies have already been performed on the liquid noble metals [45-49].

The first AIMD calculation of l-Cu was performed by Pasquarello et al. [45], who studied some static properties near melting using ultrasoft pseudopotentials [50] combined with a plane-wave expansion for the electronic orbitals. The simulation used 50 atoms, lasted for 2 ps and results were obtained for the pair distribution function, the self-diffusion coefficient and the electronic density of states. More recently, Mitrohkin [46] has performed AIMD simulations to analyze the melting process in Cu. The study used 62 atoms, lasted for 3 ps and produced results for some static properties and diffusion coefficient of l-Cu near melting. Two further AIMD studies of $\mathrm{Cu}$ [47,48] focused on the possible appearance of icosahedral 
arrangements of atoms in liquid and undercooled $\mathrm{Cu}$, with sample sizes between 100 and 200 particles and equilibrium simulation times from 1 to 5 ps. Pasturel et al. [49], within a wider study of Au-Si alloys, also performed AIMD simulations of liquid and undercooled $\mathrm{Au}$, using 256 atoms and equilibrium runs 6 ps long, and obtained results for the temperature variation of the self-diffusion coefficient and icosahedral atomic arrangements. However, none of these AIMD calculations produced results for the dynamical properties, because its evaluation requires in general larger systems, and, in particular, substantially longer simulation times.

This goal can be achieved by resorting to the orbital free ab-initio molecular dynamics (OF-AIMD) simulation method [51-57]. It is based on the Hohenberg and Kohn version of the DFT theory [43] where the electronic orbitals are replaced by the total valence electron density which now becomes the basic variable. This procedure greatly reduces the number of variables describing electronic states and, therefore, it enables one to study larger samples (a few thousand atoms) and for longer simulation times (tens of picoseconds). Now, the interaction among the positive ions and the valence electrons is characterized by means of a local pseudopotential which plays an important role in determining the ground state energy and the realistic forces acting on ions.

This paper reports an OF-AIMD study of the static and dynamic properties of the liquid noble metals $(\mathrm{Cu}, \mathrm{Ag}, \mathrm{Au})$ at thermodynamic conditions close to their respective melting points. The layout of the paper is as follows. In section 2 we briefly describe the OF-AIMD method and provide some technical details. We also describe the local ionic pseudopotentials used in this calculations. Section 3 reports and discusses the results of the ab-initio simulations for several static and dynamic properties which, moreover, are compared with the available experimental data. We conclude this paper in section 4.

\section{Theory}

A simple liquid metal is modelled as a disordered array of $N$ bare ions with valence $Z$, enclosed in a volume $V$, and interacting with $N_{\mathrm{e}}=N Z$ valence electrons through an electron-ion potential $v(r)$. The total potential energy of the system can be written, within the Born-Oppenheimer approximation, as the sum of the direct ion-ion coulombic interaction energy and the ground state energy of the electronic system under the external potential created by the ions, $V_{\text {ext }}\left(\vec{r},\left\{\vec{R}_{l}\right\}\right)=\sum_{i=1}^{N} v\left(\left|\vec{r}-\vec{R}_{i}\right|\right)$,

$$
E\left(\left\{\vec{R}_{l}\right\}\right)=\sum_{i<j} \frac{Z^{2}}{\left|\vec{R}_{i}-\vec{R}_{j}\right|}+E_{\mathrm{g}}\left[n_{\mathrm{g}}(\vec{r}), V_{\mathrm{ext}}\left(\vec{r},\left\{\vec{R}_{l}\right\}\right)\right],
$$

where $n_{\mathrm{g}}(\vec{r})$ is the ground state valence electron density and $\vec{R}_{l}$ are the ionic positions. According to DFT, the ground state valence electron density, $n_{\mathrm{g}}(\vec{r})$, can be obtained by minimizing an energy functional $E[n]$, which can be written

$$
E[n(\vec{r})]=T_{\mathrm{S}}[n]+E_{H}[n]+E_{\mathrm{Xc}}[n]+E_{\mathrm{ext}}[n],
$$

where the terms represent, respectively, the electronic kinetic energy, $T_{\mathrm{s}}[n]$, of a non-interacting system of density $n(\vec{r})$, the classical electrostatic energy (Hartree term), the exchange-correlation energy, $E_{\mathrm{xc}}[n]$, for which we used the local density approximation and finally the electron-ion interaction energy, $E_{\text {ext }}[n]$, where the electron-ion potential is characterized by a local ionic pseudopotential,

$$
E_{\text {ext }}[n]=\int \mathrm{d} \vec{r} n(\vec{r}) V_{\text {ext }}(\vec{r})
$$

For $T_{\mathrm{s}}[n]$ we used an explicit, albeit approximate, functional of the valence electron density. Several expressions were proposed and in the present calculations we used an average density model [55, 56], which provided a good description for a range of liquid simple metals, namely $T_{\mathrm{S}}[n]=T_{\mathrm{W}}[n]+T_{\alpha}[n]$, where

$$
T_{\mathrm{W}}[n(\vec{r})]=\frac{1}{8} \int \mathrm{d} \vec{r}|\nabla n(\vec{r})|^{2} / n(\vec{r})
$$


is the well-known von Weizsäcker term, and

$$
\begin{aligned}
T_{\alpha}[n] & =\frac{3}{10} \int \mathrm{d} \vec{r}[n(\vec{r})]^{5 / 3-2 \alpha}[\tilde{k}(\vec{r})]^{2}, \\
\tilde{k}(\vec{r}) & =\left(2 k_{\mathrm{F}}^{0}\right)^{3} \int \mathrm{d} \vec{s} k(\vec{s}) w_{\alpha}\left(2 k_{F}^{0}|\vec{r}-\vec{s}|\right),
\end{aligned}
$$

where $k(\vec{r})=\left(3 \pi^{2}\right)^{1 / 3}[n(\vec{r})]^{\alpha}, k_{\mathrm{F}}^{0}$ is the Fermi wavevector for a mean electron density $n_{\mathrm{e}}=N_{\mathrm{e}} / V$, and $w_{\alpha}(x)$ is a weight function chosen so that both the linear response theory and Thomas-Fermi limits are correctly recovered. Further details are given in reference [55, 56].

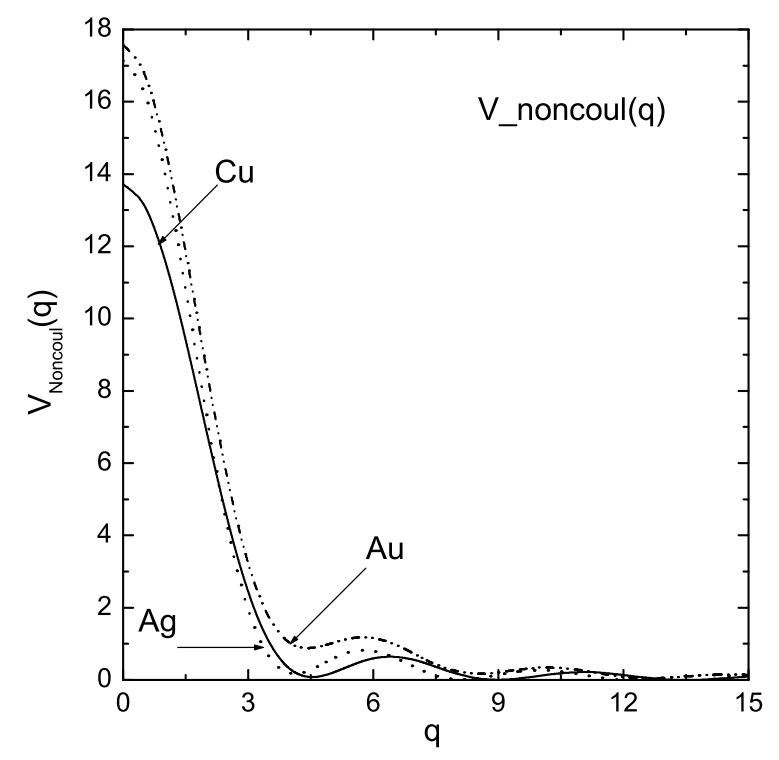

Figure 1. Non-Coulombic part of the electron-ion interaction for liquid $\mathrm{Cu}, \mathrm{Ag}$ and $\mathrm{Au}$.

Another basic ingredient in the above formalism, is the local ionic pseudopotential, $v_{\mathrm{ps}}(r)$, that describes the ion-electron interaction. The AIMD simulations based on KS-AIMD method usually employ non-local pseudopotentials [58] obtained by fitting to some properties of the free atom [59_61]. However, in the present OF-AIMD approach, the valence electron density is the basic variable, and non-local pseudopotentials cannot be used. Therefore, the interaction among the valence electrons and the ions must be described using a local pseudopotential which is usually chosen so as to include an accurate description of the electronic structure in the physical state of interest. Bhuiyan et al. [57] developed a local pseudopotential model which in conjunction with the OF-AIMD method has provided a good description of several static and dynamic properties of l-Sn near melting [57]. Specifically, it is defined as

$$
\nu_{\mathrm{ps}}(r)=\left\{\begin{aligned}
A+B \exp (-r / a), & r<R_{\mathrm{C}}, \\
-Z / r, & r>R_{\mathrm{C}},
\end{aligned}\right.
$$

where $A$ and $B$ are contants, $R_{\mathrm{C}}$ is a core radius and $a$ is the softness parameter. Aiming to reduce the number of free parameters, we impose the condition that the logarithmic derivatives of the potential inside and outside the core are exactly the same at the core radius. This permits to eliminate $B$ as a parameter, and the successfulness of this approach can be judged by the capability of recovering the available experimental data. The other parameters $A, a$ and $R_{C}$, and the effective valence $Z$, have been chosen so that the OF-AIMD simulation reproduces the experimental static structure factor. The values obtained herein are given in table 1 where we notice that for a given system the parameters remain constant for both thermodynamic states. In figure 1 we depicted the non-Coulombic part of the ionic pseudopotential for $\mathrm{l}-\mathrm{Cu}, \mathrm{l}-\mathrm{Ag}$ and $\mathrm{l}-\mathrm{Au}$. It shows that in the long wavelength limit $(q \rightarrow 0)$, the value of 
Table 1. Input parameters used in the calculations; temperature $T$, ionic number density $\rho$, amplitude in the core $A$, softness parameter $a$, core radius $R_{\mathrm{C}}$ and the effective ionic valence $Z$.

\begin{tabular}{|c|l|l|l|l|l|l|}
\hline System & $T(\mathrm{~K})$ & $\rho\left(\AA^{-3}\right)$ & $A(\mathrm{au})$ & $a(\mathrm{au})$ & $R_{\mathrm{C}}(\mathrm{au})$ & $Z$ \\
\hline \hline $\mathrm{Cu}$ & 1423 & 0.0755 & 0.05 & 0.3 & 1.40 & 1.35 \\
& 1773 & 0.0728 & 0.05 & 0.3 & 1.40 & 1.35 \\
\hline $\mathrm{Ag}$ & 1273 & 0.0517 & 0.05 & 0.3 & 1.55 & 1.35 \\
& 1673 & 0.0496 & 0.05 & 0.3 & 1.55 & 1.35 \\
\hline $\mathrm{Au}$ & 1423 & 0.0525 & 0.05 & 0.2 & 1.50 & 1.35 \\
& 1773 & 0.0517 & 0.05 & 0.2 & 1.50 & 1.35 \\
\hline
\end{tabular}

$v_{\mathrm{ps}}(q)$ is the largest for $\mathrm{l}-\mathrm{Au}$ and the smallest for $\mathrm{l}-\mathrm{Cu}$. Note also that the phase of oscillations is different for each system.

We stress that the combination of the OF-AIMD method with local ionic pseudopotentials has already provided accurate descriptions of several static and dynamic properties for a range of bulk liquid simple metals and binary alloys [55, 56, 62-64].

\section{Results and discussion}

OF-AIMD simulations have been performed for $\mathrm{l}-\mathrm{Cu}, \mathrm{l}-\mathrm{Ag}$ and $\mathrm{l}-\mathrm{Au}$ at two thermodynamic states near their respective triple points. Those states were chosen due to the availability of experimental XR diffraction data [24]. Table 1 gives additional information about thermodynamic states and other input parameters used for the simulation.

The simulations were carried out using 500 particles in a cubic cell with periodic boundary conditions and whose size was appropriate for the corresponding experimental ionic number density. Given the ionic positions at time $t$, the electronic energy functional is minimized with respect to $n(\vec{r})$ represented by a single effective orbital, $\psi(\vec{r})$, defined as $n(\vec{r})=[\psi(\vec{r})]^{2}$. The orbital is expanded in plane waves which are truncated at a cutoff energy, $E_{\text {Cut }}=20.0$ Ryd. The energy minimization with respect to the Fourier coefficients of the expansion is performed every ionic time step using a quenching method which results in the ground state valence electron density and energy. The forces on the ions are obtained from the electronic ground state via the Hellman-Feynman theorem, and the ionic positions and velocities are updated by solving Newton's equations, using the Verlet leapfrog algorithm with a timestep of $6.0 \cdot 10^{-3} \mathrm{ps}$. Equilibration in the simulations lasted $10 \mathrm{ps}$. and the calculation of properties was made by averaging over 150 ps.

In this study, we have evaluated several liquid static properties (pair distribution function and static structure factor) as well as various dynamic properties, both single-particle ones (velocity autocorrelation function, mean square displacement) and collective ones (intermediate scattering functions, dynamic structure factors, longitudinal and transverse currents). The calculation of the time correlation functions (CF) was performed by taking time origins every five time steps. Several CF have also a dependence on the wave vectors $\vec{q}$ which depend only on $q \equiv|\vec{q}|$ because our system is isotropic.

\subsection{Static Properties}

\subsubsection{Liquid $\mathrm{Cu}$}

The OF-AIMD simulation permits to directly evaluate the static structure factor, $S(q)$, and its real space counterpart, i.e., the pair distribution function $g(r)$. Figure 2 (a) shows the calculated $S(q)$ for l-Cu at two different thermodynamic states characterized by temperatures $T=1423$ and $1773 \mathrm{~K}$. For both states, the main peak is located at $q_{\mathrm{p}} \approx 2.88 \AA^{-1}$. Comparison with the XR data [24] shows an overall good agreement for both the positions and phases of the oscillations, although the present OF-AIMD results slightly overestimate the height of the main peak. Note, however, that the height of the main peak in 

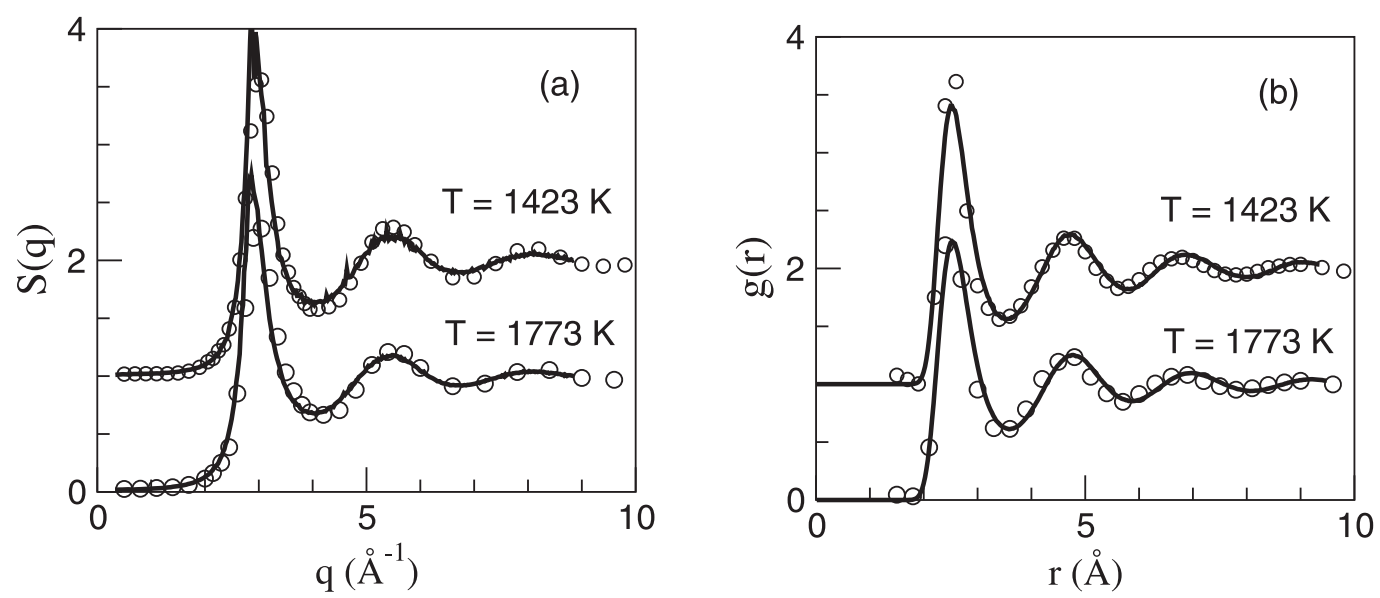

Figure 2. (a) Static structure factors and (b) pair correlation functions for l-Cu at two thermodynamic states. Solid lines are the OF-AIMD results and the open circles stand for the XR diffraction data.

the neutron data of Eder et al. at $1393 \mathrm{~K}$ (not shown) is substantially higher than in the XR data, being in better agreement with our results. A similar overestimation of the height of the main peak of $S(q)$ in l-Cu, as compared to XR measurements, was also obtained in CMD studies carried out using EAM-based potentials [17, 38, 65]. The KS-AIMD of Ganesh and Widom [47] at $1398 \mathrm{~K}$ also yield a structure factor with a height of the main peak similar to our data and to the neutron measurements. The agreement of our high temperature results with experiment is the same, while in this case, the XR structure factor at $1773 \mathrm{~K}$ and the corresponding neutron data at $1833 \mathrm{~K}$ agree better with each other than at lower temperatures.

The long wavelength limit of the static structure factor, $S(q \rightarrow 0)$, is linked with thermodynamics through the relationship $S(q \rightarrow 0)=\rho k_{\mathrm{B}} T \kappa_{\mathrm{T}}$ where $k_{\mathrm{B}}$ is Boltzmann's constant and $\kappa_{\mathrm{T}}$ is isothermal compressibility. A least squares fit of $S(q)=s_{0}+s_{2} q^{2}+s_{4} q^{4}$ to the calculated $S(q)$ for small $q$-values yields an estimate $\kappa_{T, \mathrm{OF}-\mathrm{AIMD}}=0.90 \pm 0.03$ (in units of $10^{-11} \mathrm{~N}^{-1} \mathrm{~m}^{2}$ ) for $T=1423 \mathrm{~K}$, underestimating the experimental value of 1.49 [27, 66], or 1.41 [28]. For $T=1773 \mathrm{~K}$ we find $\kappa_{\mathrm{T}}=1.09 \pm 0.03$, while the experimental value is 1.74 (in the same units) [28].

The calculated pair distribution functions, $g(r)$, are depicted in figure 2(b) along with the corresponding XR data [24]. The main peak is located at $r_{\mathrm{p}}=2.53 \AA$ and $2.55 \AA$ for $T=1443$ and $1773 \mathrm{~K}$, respectively, which agrees with the corresponding experimental data. A similar good agreement is found for the positions and the phase of oscillations of the subsequent peaks. The only noticeable discrepancy concerns the height of the main peak which is slightly underestimated by the present calculations. Nevertheless, we note that a similar disparity is also reported in KS-AIMD studies [45, 47, 48]. The average number of nearest neighbors, also known as coordination number $(\mathrm{CN})$, is obtained by integrating the radial distribution function (RDF), $4 \pi r^{2} \rho g(r)$, up to a distance $r_{m}$ which is usually identified as the position of the first minimum in either the RDF or the $g(r)$ [67,68]. Both choices often lead to rather similar results and in what follows we report the results obtained by integrating up to the first minimum of the RDF which was found at $r_{\mathrm{m}} \approx 3.42$ and $3.44 \AA$ for $T=1443$ and $1773 \mathrm{~K}$, leading to values $\mathrm{CN} \approx 12.9$ and 12.6 , respectively. For comparison, we note that the KS-AIMD studies at $1500 \mathrm{~K}$ produce $\mathrm{CN} \approx 12.5$ [45], 12.3 [47], and 12.9 [48] using a bit different integration limits.

\subsubsection{Liquid $\mathrm{Ag}$}

The calculated $S(q)$ for l-Ag at two different states are plotted in figure 3 (a) where they are compared with the corresponding XR data [24]. The calculated position of the main peak are at $q_{\mathrm{p}}=2.57$ and $2.59 \AA^{-1}$ for $T=1273 \mathrm{~K}$ and $1673 \mathrm{~K}$, respectively. For a lower temperature, $T=1273 \mathrm{~K}$, we observe that the calculated height of the main peak is a bit bigger than that of the XR data [24]; indeed, a similar disparity has also been reported in other CMD studies for l-Ag [17, 38]. Note also that the neutron $S(q)$ of Bellisent et al. at $1323 \mathrm{~K}$ (not shown) has the height of the main peak of 2.85, which is much higher than Waseda's data, and is more in line with our result. On the other hand, the positions and phase of 

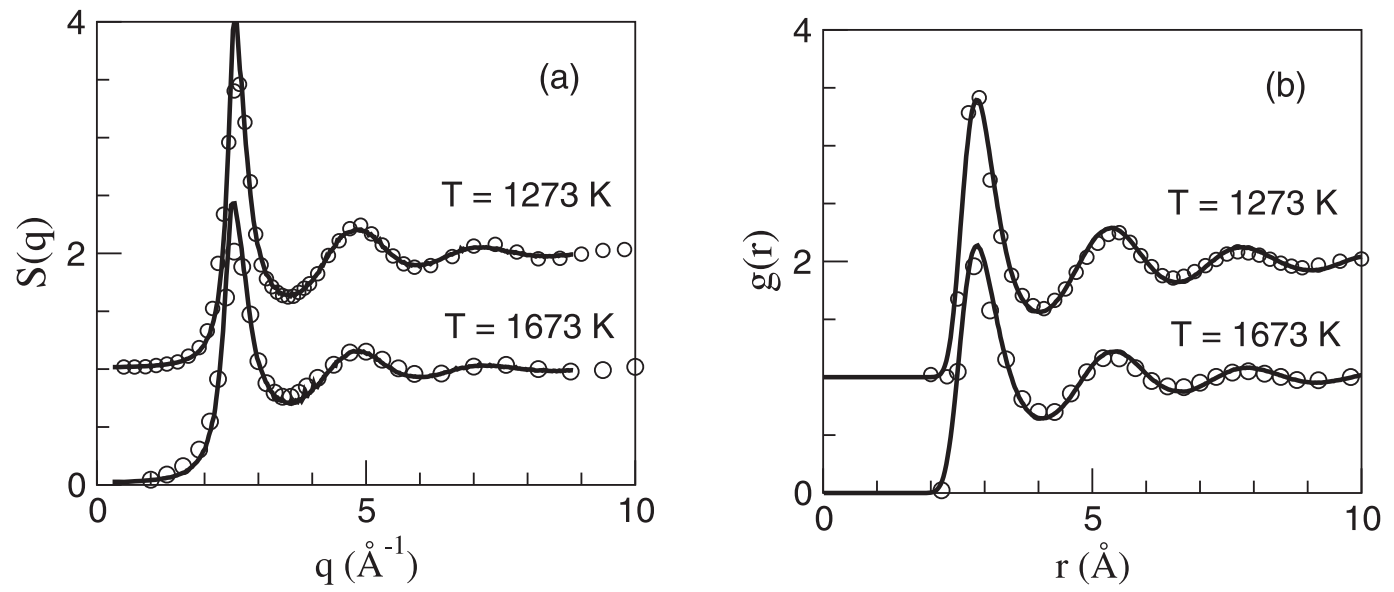

Figure 3. (a) Static structure factors and (b) pair correlation functions for l-Ag at two thermodynamic states. Solid lines are the OF-AIMD results and the open circles are the XR diffraction data.

oscillations of the subsequent peaks are found to be in very good agreement with experiment. We have also calculated the isothermal compressibility of $\mathrm{l}-\mathrm{Ag}$ at $T=1273 \mathrm{~K}$ and we have obtained $\kappa_{\mathrm{T}}=1.94 \pm 0.08$ (in units of $10^{-11} \mathrm{~N}^{-1} \mathrm{~m}^{2}$ ) to be compared with the experimental data of 2.11 [66], 1.92 [27], or 1.80 [28]. For $T=1673 \mathrm{~K}$, we have obtained $\kappa_{\mathrm{T}}=2.19 \pm 0.05$, while experiment yields 2.21 [28].

The calculated pair correlation functions, $g(r)$, for l-Ag are depicted in figure 3(b) for $T=1273 \mathrm{~K}$ and $1673 \mathrm{~K}$ where we observe a good agreement with the respective XR data [24]. Integrating up to the first minima of the RDF, found at $r_{\mathrm{m}}=3.86 \AA$ and $3.82 \AA$ for $T=1273$ and $1673 \mathrm{~K}$, respectively, we obtain the values $\mathrm{CN} \approx 12.6$ and 11.7 , respectively.

\subsubsection{Liquid Au}

The calculated $S(q)$ for l-Au at $T=1423$ and $1773 \mathrm{~K}$ are depicted in figure 4(a) along with the corresponding XR data [24]. For both states, the main peak is located at $q_{\mathrm{p}}=2.60 \AA^{-1}$ and a good agreement with experiment is observed for the positions and magnitudes of the main and subsequent peaks. The calculated isothermal compressibility has yielded values $\kappa_{\mathrm{T}}=1.61 \pm 0.07$ (in units of $10^{-11} \mathrm{~N}^{-1} \mathrm{~m}^{2}$ ) at $1423 \mathrm{~K}$, to compare with 1.31 [27] or 1.27 [28], and $\kappa_{\mathrm{T}}=2.06 \pm 0.06$ at $1773 \mathrm{~K}$, where Singh et al. report 1.61 [28].

The $g(r)$ for l-Au at $T=1423 \mathrm{~K}$ and $1773 \mathrm{~K}$ are depicted in figure 4 (b). For both states, the main peak is located at $r_{\mathrm{p}}=2.80 \AA$, which coincides with the experimental value, although the height of the main
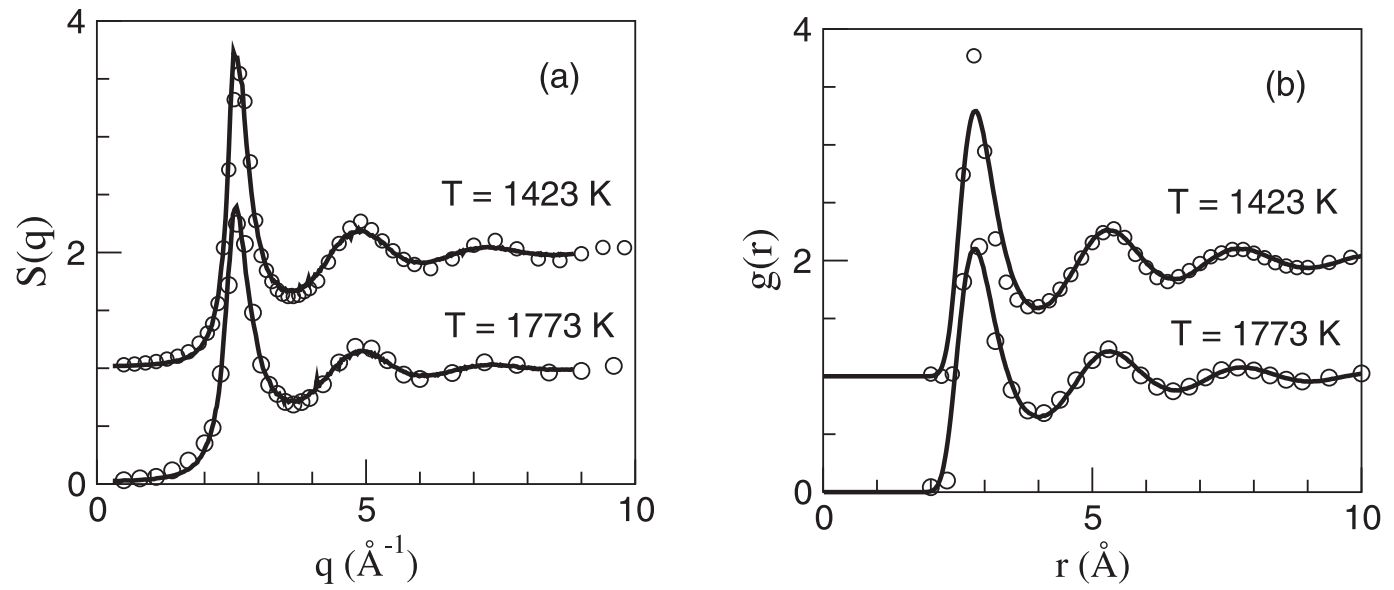

Figure 4. (a) Static structure factors and (b) pair correlation functions for l-Au at two thermodynamic states. Solid lines are the OF-AIMD results and the open circles are the XR diffraction data. 
peak is somewhat underestimated, especially for the lower temperature. The RDF has a first minimum at $r_{\mathrm{m}}=3.86 \AA$ and $3.82 \AA$ which yields values of $\mathrm{CN} \approx 12.7$ and 12.2 for $T=1423 \mathrm{~K}$ and $1773 \mathrm{~K}$, respectively.

\subsection{Dynamic properties: Single particle dynamics}

Relevant information concerning the single particle dynamics can be derived from several magnitudes and here we report our results obtained for some of those magnitudes.

The self-intermediate scattering function, $F_{\mathrm{s}}(q, t)$, provides a detailed information on the single particle dynamic properties over different length scales going from hydrodynamic $(q \rightarrow 0)$ to free particle $(q \rightarrow \infty)$ limits. This is defined as

$$
F_{\mathrm{s}}(q, t)=\frac{1}{N}\left\langle\sum_{j=1}^{N} \exp \left[\mathrm{i} \vec{q} \vec{R}_{j}\left(t+t_{0}\right)\right] \exp \left[-\mathrm{i} \vec{q} \vec{R}_{j}\left(t_{0}\right)\right]\right\rangle,
$$

where $\langle\ldots\rangle$ denotes the average over time origins and wavevectors with the same module. Closely connected to the $F_{\mathrm{s}}(q, t)$, is the velocity autocorrelation function (VACF) of a tagged ion in the fluid, $Z(t)$, which can be obtained as the $q \rightarrow 0$ limit of the first-order memory function of the $F_{\mathrm{S}}(q, t)$ although in the present simulations it was calculated from its definition

$$
Z(t)=\left\langle\vec{v}_{1}(t) \vec{v}_{1}(0)\right\rangle /\left\langle v_{1}^{2}\right\rangle,
$$

which stands for the normalized VACF. It provides information on the motion of an atom inside the cage created by the shell of nearest neighbors. Besides, its time integral leads to the self-diffusion coefficient, $D$, namely

$$
D=\frac{1}{\beta m} \int_{0}^{\infty} Z(t) \mathrm{d} t
$$

where $\beta=1 /\left(k_{\mathrm{B}} T\right) . D$ can also be obtained from the slope of the mean square displacement $\delta R^{2}(t)$ of a tagged ion in the fluid, as

$$
D=\lim _{t \rightarrow \infty} \delta R^{2}(t) / 6 t=\lim _{t \rightarrow \infty} \frac{1}{6} \frac{\mathrm{d} \delta R^{2}(t)}{\mathrm{d} t}, \quad \delta R^{2}(t) \equiv\left\langle\left|\vec{R}_{1}(t)-\vec{R}_{1}(0)\right|^{2}\right\rangle .
$$

In the present OF-AIMD calculations, both routes have led to practically the same $D$ value.

\subsubsection{Liquid $\mathrm{Cu}$}

Figure 5 (a) shows, for several $q$-values, the calculated $F_{\mathrm{s}}(q, t)$ for $\mathrm{l}-\mathrm{Cu}$ at $T=1423 \mathrm{~K}$. We observe the typical monotonous, non-linear decrease with time which becomes faster with increasing $q$-values; moreover, comparison with the simple liquid metals near their respective melting points shows that at similar $q / q_{\mathrm{p}}$ values, the $F_{\mathrm{s}}(q, t)$ has a comparable rate of decay [55, 56, 69- 72].

The calculated $Z(t)$ for l-Cu are shown in figure 5(b). The main features in the $Z(t)$ are comparable to those obtained for simple liquid metals near melting [55, 56, 69], namely a first minimum about 0.30 deep and a subsequent maximum with a rather weak amplitude. We recall that the negative values of $Z(t)$ represent a backscattering effect induced by the cage effect; moreover, with increasing temperature (and decreasing density) the cage effect becomes less relevant, i.e., the first minimum in $Z(t)$ is shallower while the subsequent oscillations are less marked.

The self-diffusion coefficient was calculated according to equations (7)-8), leading to values $D=$ $0.39 \AA^{2} / \mathrm{ps}(T=1423 \mathrm{~K})$ and $0.58 \AA^{2} / \mathrm{ps}(T=1773 \mathrm{~K})$. The reference experimental value at $T=1423 \mathrm{~K}$ is $0.40 \AA^{2} / \mathrm{ps}$ [30, 31], while the recent measurements of Meyer yielded $D=0.37 \AA^{2} / \mathrm{ps}$ at $1420 \mathrm{~K}$, which are both in excellent agreement with our estimate. Other CMD studies yielded the values $D=0.31$ and $0.27 \AA^{2} / \mathrm{ps}$ [38, 39] and $D=0.36 \AA^{2} / \mathrm{ps}$ for $T=1400 \mathrm{~K}$ [40]. The AIMD studies at $1500 \mathrm{~K}$ produced diffusion coefficients of $D=0.28$ [45], which clearly underestimates the experimental data, presumably due to a small number of particles (50 atoms) used in the simulation, and $0.40 \pm 0.05$ [48] in good agreement with experiment. The higher temperature is outside the range of measurements by Meyer [32] (up to $1620 \mathrm{~K}$ ). 

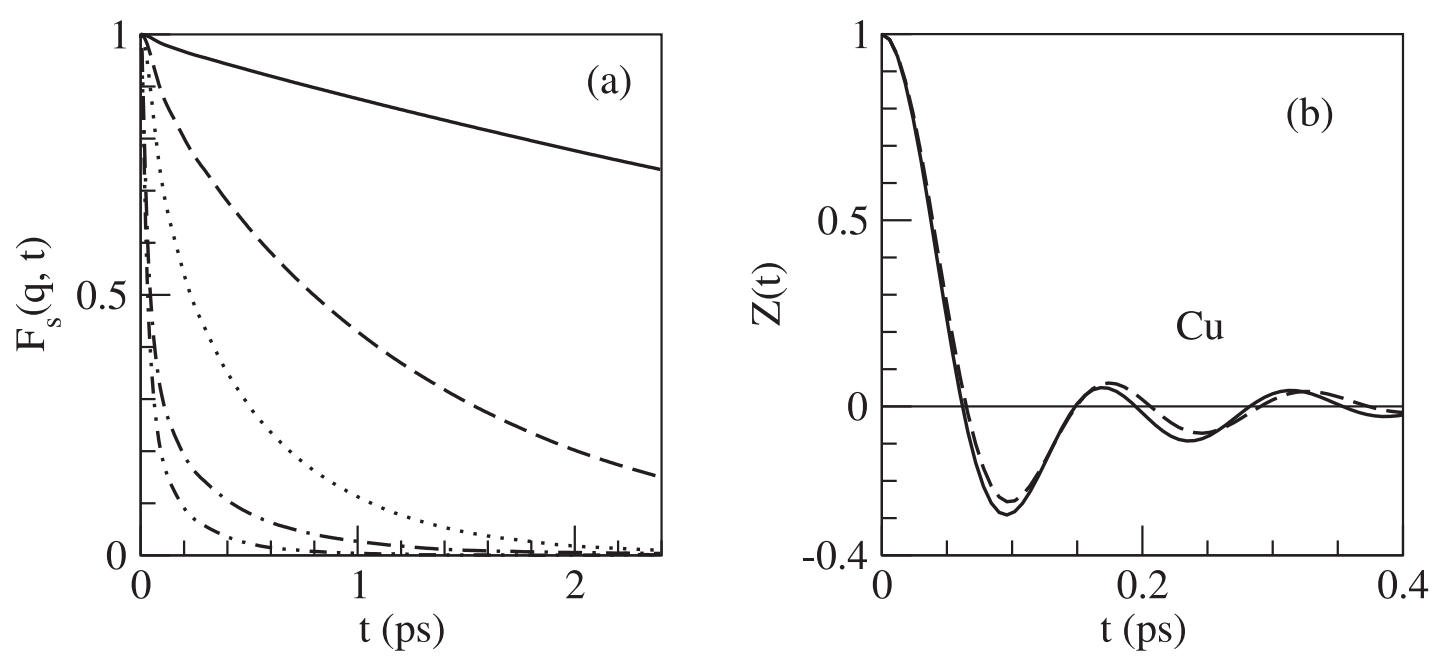

Figure 5. (a) Self-intermediate scattering function of l-Cu at $T=1423 \mathrm{~K}$. Full line: $0.6 \AA^{-1}$, dashed line: $1.5 \AA^{-1}$, dotted line: $2.5 \AA^{-1}$, dotted-dashed line: $3.1 \AA^{-1}$ and double dotted-dashed line: $4.3 \AA^{-1}$ (b) Normalized velocity autocorrelation function for l-Cu at $1423 \mathrm{~K}$ (full line) and $1773 \mathrm{~K}$ (dashed line).

However, he found that the measured data could be well described through an Arrhenius formula. The value obtained with this expression for $T=1773 \mathrm{~K}$ is $D=0.65 \pm 0.05 \AA^{2} / \mathrm{ps}$, which is in reasonable agreement with our result. For this temperature, the CMD study of Han et al. [40] has reported $D=0.59 \AA^{2} / \mathrm{ps}$ for $T=1700 \mathrm{~K}$, which is also similar to our present estimate.

\subsubsection{Liquid Ag}

Figure 6 (a) shows the calculated $F_{\mathrm{s}}(q, t)$, at several $q$-values, for l-Ag at $T=1273 \mathrm{~K}$ and we observe the features very similar to those already found in $\mathrm{l}-\mathrm{Cu}$. The normalized VACF for $\mathrm{l}-\mathrm{Ag}$ at $T=1273$ and $1673 \mathrm{~K}$ are depicted in figure 6(b) where we observe the typical cage effect; now the variation with temperature is more marked than in l-Cu because the relative change in the ionic density is greater. Now the $Z(t)$ of l-Ag becomes negative for longer times and this is because the backscattering associated with the cage effect in l-Ag is reduced by the combination of two factors, namely, a smaller ionic number density
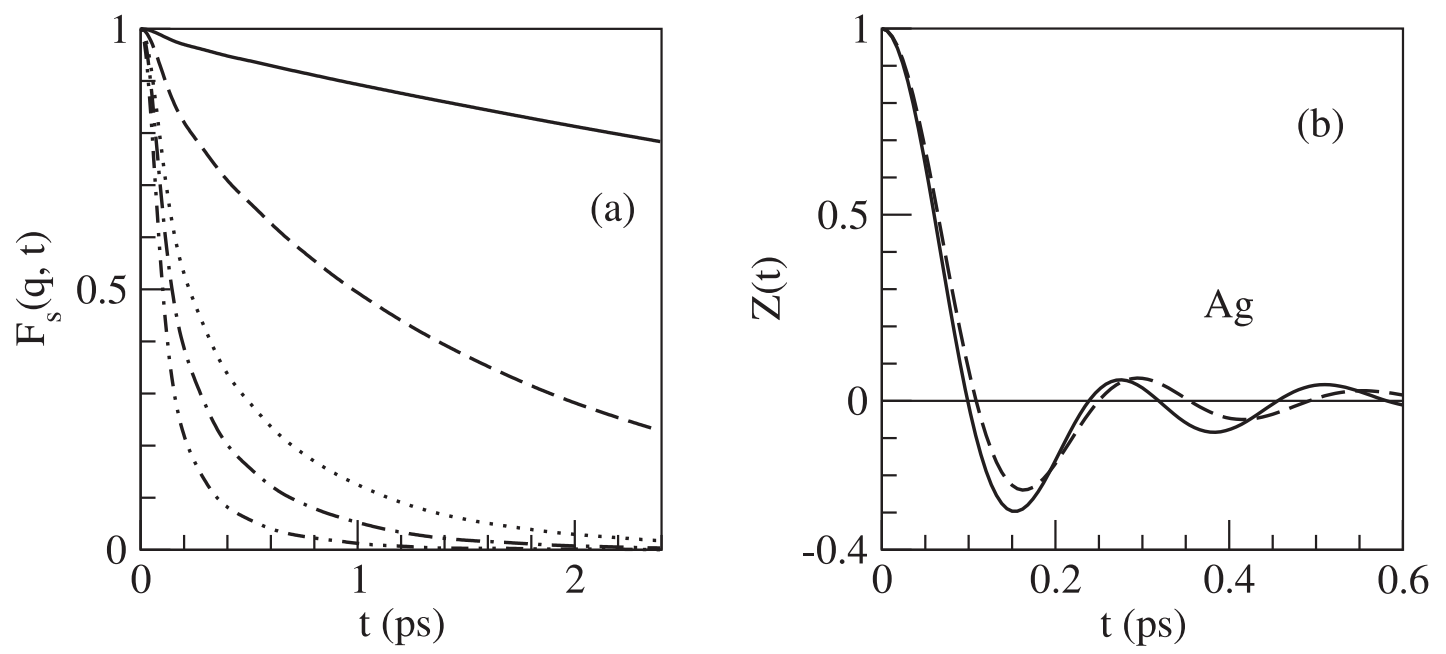

Figure 6. (a) Self-intermediate scattering function of l-Ag at $T=1273 \mathrm{~K}$. Full line: $0.59 \AA^{-1}$, dashed line: $1.5 \AA^{-1}$, dotted line: $2.7 \AA^{-1}$, dotted-dashed line: $3.3 \AA^{-1}$ and double dotted-dashed line: $4.2 \AA^{-1}(\mathrm{~b})$ normalized velocity autocorrelation function for l-Ag at $1273 \mathrm{~K}$ (full line) and $1673 \mathrm{~K}$ (dashed line). 
and a greater atomic mass. The calculated self-diffusion coefficients are $D=0.29 \AA^{2} / \mathrm{ps}$ and $0.55 \AA^{2} / \mathrm{ps}$ for $T=1273 \mathrm{~K}$ and $1673 \mathrm{~K}$, respectively, which is very close to the experimental data of $D=0.28 \AA^{2} / \mathrm{ps}$ and $0.58 \AA^{2} / \mathrm{ps}[30]$

\subsubsection{Liquid Au}

The calculated $F_{\mathrm{S}}(q, t)$, at several $q$-values, for l-Au at $T=1423 \mathrm{~K}$ is depicted in figure $7(\mathrm{a})$. As for the normalized VACF, figure 7 (b) shows the calculated $Z(t)$ for $T=1423$ and $1773 \mathrm{~K}$. Notice that in comparison with the previous results for l-Ag, the $Z(t)$ for l-Au take longer to become negative and this is due to a weaker backscattering effect induced by the greater atomic mass of the Au ions. Comparison with the $Z(t)$ of Bogicevic et al. [35] shows that these authors obtain a $Z(t)$ with a narrower and shallower first minimum along with weaker oscillations. The calculated self-diffusion coefficients are $D=0.27$ and $0.46 \AA^{2} / \mathrm{ps}$
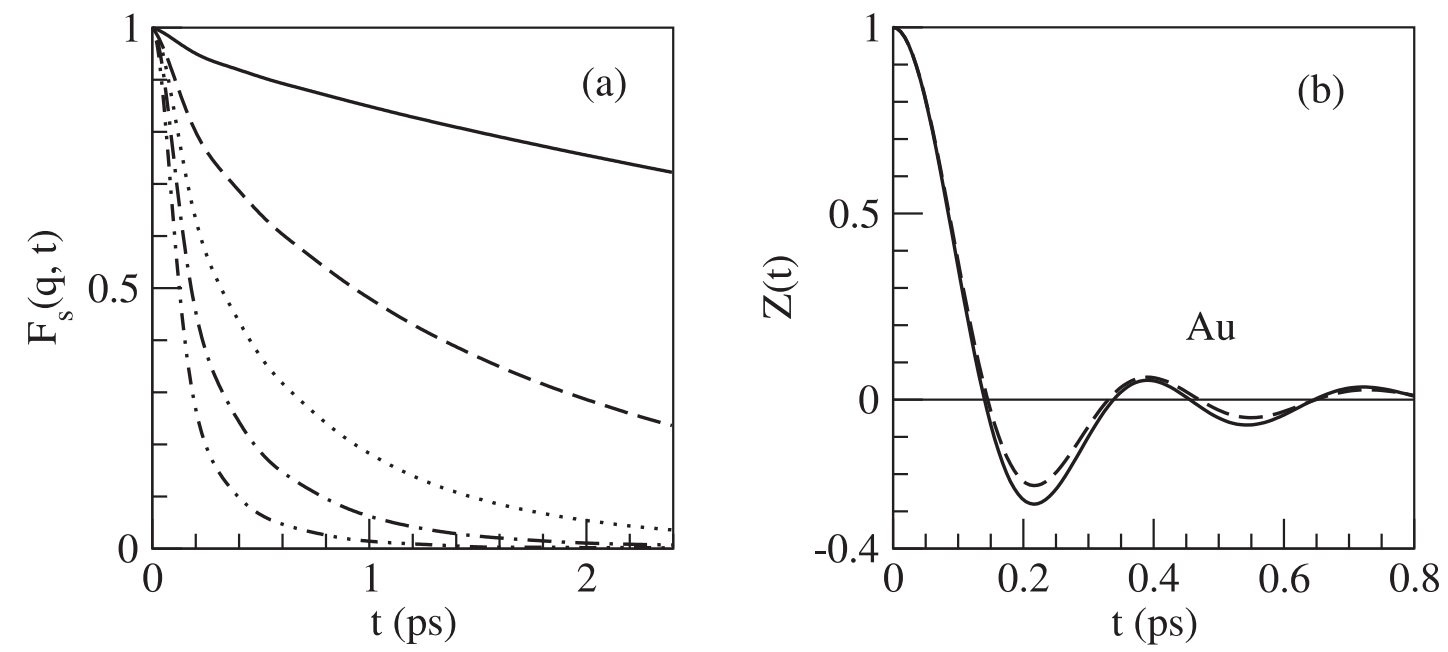

Figure 7. (a) Self-intermediate scattering function of l-Au at $T=1473 \mathrm{~K}$. Full line: $0.59 \AA^{-1}$, dashed line: $1.39 \AA^{-1}$, dotted line: $2.41 \AA^{-1}$, dotted-dashed line: $3.2 \AA^{-1}$ and double dotted-dashed line: $4.2 \AA^{-1}$ (b) normalized velocity autocorrelation function for l-Au at $1423 \mathrm{~K}$ (full line) and $1773 \mathrm{~K}$ (dashed line).

at $T=1423$ and $1773 \mathrm{~K}$, respectively, to be compared with an experimental value [66] of $0.24 \AA^{2} / \mathrm{ps}$ for l-Au at $T=1423 \mathrm{~K}$. The calculations of Bogicevic et al. [35] yielded somewhat bigger values, namely $D=0.31$ and $0.60 \AA^{2} / \mathrm{ps}$ at $T=1423$ and $1773 \mathrm{~K}$, respectively, whose origin can be traced back to the less marked cage effect in their $Z(t)$. On the other hand, we mention that CMD simulations using two different EAM potentials for l-Au at $T=1423 \mathrm{~K}$ yielded $D=0.26 \AA^{2} / \mathrm{ps}$ [43,48]. The AIMD simulations of Pasturel et al. [49] produced a value of $D=0.153 \AA^{2} / \mathrm{ps}$ at $1400 \mathrm{~K}$, somewhat smaller than experiment, and $0.30 \AA^{2} / \mathrm{ps}$ at $1700 \mathrm{~K}$. To our knowledge, no experimental data are availble for the self-diffusion coefficient of l-Au at $T=1773 \mathrm{~K}$.

\subsection{Dynamic properties: Collective dynamics}

Regarding the collective dynamics, the most important magnitude is the intermediate scattering function, $F(q, t)$, which provides information on the collective dynamics of density fluctuations. It is defined as

$$
F(q, t)=\frac{1}{N}\left\langle\sum_{j=1}^{N} \exp \left[\mathrm{i} \vec{q} \vec{R}_{j}\left(t+t_{0}\right)\right] \sum_{l=1}^{N} \exp \left[-\mathrm{i} \vec{q} \vec{R}_{l}\left(t_{0}\right)\right]\right\rangle .
$$

Its space Fourier transform (FT) produces the van Hove correlation function whereas its time FT results in the dynamic structure factor, $S(q, \omega)$, which is the magnitude measured in the inelastic XR (or neutron) scattering experiments. 
Another interesting magnitude associated with the density fluctuations is current due to the overall motion of particles, i.e.,

$$
\vec{j}(q, t)=\sum_{j=1}^{N} \vec{v}_{j}(t) \exp \left[\mathrm{i} \vec{q} \cdot \vec{R}_{j}(t)\right],
$$

which is usually split into a longitudinal component, $\vec{j}_{\mathrm{L}}(q, t)$ parallel to $\vec{q}$, and a transverse component, $\vec{j}_{\mathrm{T}}(q, t)$, perpendicular to $\vec{q}$. Therefrom, the longitudinal, $J_{\mathrm{L}}(q, t)$, and transverse $J_{\mathrm{T}}(q, t)$, current correlation functions are obtained as

$$
J_{\mathrm{L}}(q, t)=\frac{1}{N}\left\langle\vec{j}_{\mathrm{L}}(q, t) \cdot \vec{j}_{\mathrm{L}}^{*}(q, 0)\right\rangle, \quad J_{\mathrm{T}}(q, t)=\frac{1}{2 N}\left\langle\vec{j}_{\mathrm{T}}(q, t) \cdot \vec{j}_{\mathrm{T}}^{*}(q, 0)\right\rangle .
$$

The corresponding time FT produce the associated spectra, $J_{\mathrm{L}}(q, \omega)$ and $J_{\mathrm{T}}(q, \omega)$, respectively, with $J_{\mathrm{L}}(q, \omega)=\omega^{2} S(q, \omega)$. The transverse current correlation function $J_{\mathrm{T}}(q, t)$, is not associated with any measurable quantity and can only be determined by means of MD simulations. It provides information on the shear modes, and its shape evolves from a Gaussian, in both $q$ and $t$, for the free particle limit towards a Gaussian in $q$ and exponential in $t$ for the hydrodynamic limit $(q \rightarrow 0)$, namely

$$
J_{\mathrm{T}}(q \rightarrow 0, t)=\frac{1}{\beta m} \exp \left[-q^{2} \eta|t| /(m \rho)\right],
$$

where $\eta$ is the shear viscosity. On the other hand, for intermediate $q$-values, the $J_{\mathrm{T}}(q, t)$ shows a complicated behaviour, because it may oscillate signaling the propagation of shear waves. From the calculated $J_{\mathrm{T}}(q, t)$ it is possible to obtain the shear viscosity coefficient $\eta$ as follows. The memory function representation of $J_{\mathrm{T}}(q, t)$,

$$
\widetilde{J}_{\mathrm{T}}(q, z)=\frac{1}{\beta m}\left[z+\frac{q^{2}}{\rho m} \widetilde{\eta}(q, z)\right]^{-1},
$$

where the tilde denotes the Laplace transform, introduces a generalized shear viscosity coefficient $\widetilde{\eta}(q, z)$. The area under the normalized $J_{\mathrm{T}}(q, t)$ gives $\beta m \widetilde{J}_{\mathrm{T}}(q, z=0)$, from which $\widetilde{\eta}(q, z=0) \equiv \widetilde{\eta}(q)$ can be obtained and when extrapolated to $q \rightarrow 0$ it produces a usual shear viscosity coefficient $\eta$. This is performed [73] by exploiting the property that inversion is a symmetry in the system and, therefore, $\widetilde{\eta}(q)$ should be an even function of $q$ which permits to approximate (when $q \rightarrow 0) \widetilde{\eta}(q)=\eta\left(1-\alpha q^{2}\right.$ ).

\subsubsection{Liquid $\mathrm{Cu}$}

Figure 8 (a) shows the calculated $F(q, t) / F(q, t=0)$ at several $q$-values for $\mathrm{l}-\mathrm{Cu}$ at $T=1423 \mathrm{~K}$. The $F(q, t)$ exhibit an oscillatory behaviour at low $q$-values with the oscillations becoming weaker for increasing $q$ 's until they finally disappear at $q \approx 2.2 \AA^{-1} \approx 0.75 q_{\mathrm{p}}$. We stress that this behaviour is very similar to the one found for the simple liquid metals near melting [55, 56, 69].

From the calculated $F(q, t)$, we performed a time FT (with an appropriate window to smooth out the truncation effects) leading to the associated dynamic structure factor, $S(q, \omega)$. The obtained results are depicted in figure 8 (b) for several $q$-values. We notice that up to $q \approx 0.75 q_{\mathrm{p}}$, the calculated $S(q, \omega)$ exhibit well defined side-peaks which are indicative of collective density excitations. The FT of the longitudinal current correlation function, $J_{\mathrm{L}}(q, \omega)$, shows, however, side-peaks for all wavevectors, and from the positions of these side-peaks, $\omega_{\mathrm{L}}(q)$, a dispersion relation of the density fluctuations was obtained and plotted in figure 9 In the hydrodynamic region (small $q$ ) the slope of the dispersion relation curve is the $q$ dependent adiabatic sound velocity $c_{\mathrm{S}}(q)=v_{\text {th }} \sqrt{\gamma / S(q)}$, with $v_{\text {th }}=\sqrt{k_{\mathrm{B}} T / m}$ being the thermal velocity, $\gamma$ the ratio of specific heats and $k_{\mathrm{B}}$ Boltzmann's constant. In the $q \rightarrow 0$ limit, the $c_{\mathrm{s}}(q)$ reduces to the bulk adiabatic sound velocity $c_{\mathrm{s}}$. Using the smallest $q$-value achieved by the simulations, $q_{\mathrm{min}}=0.334 \AA^{-1}$, we get an estimate $c_{\mathrm{s}}\left(q_{\mathrm{min}}\right) \approx 3880 \mathrm{~m} / \mathrm{s}$, which is clearly above the experimental hydrodynamic value $c_{\mathrm{s}} \approx$ 3481 [27] or $3449 \mathrm{~m} / \mathrm{s}$ [28]. In figure 10 we have plotted the $c_{\mathrm{s}}(q)$ which clearly points to the existence of some positive dispersion in l-Cu. This behaviour qualitatively agrees with an estimate of $4230 \mathrm{~m} / \mathrm{s}$ [74] obtained with inelastic XR scattering data. For the higher temperature $T=1773 \mathrm{~K}$, we get a smaller value $c_{\mathrm{s}} \approx 3802 \mathrm{~m} / \mathrm{s}$, whereas the experimental hydrodynamic sound velocity is $3266 \mathrm{~m} / \mathrm{s}$ [28], signaling the persistence of the positive dispersion at these higher temperatures. 


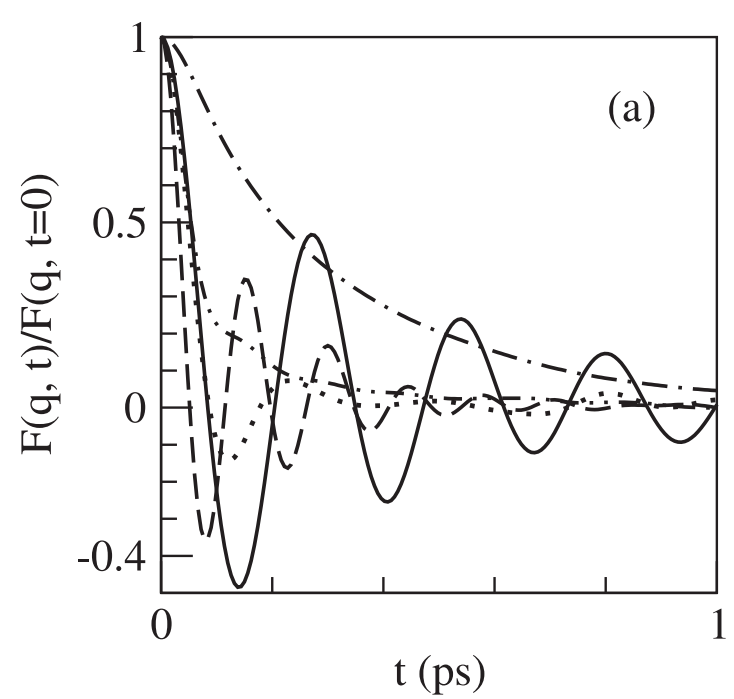

(a)

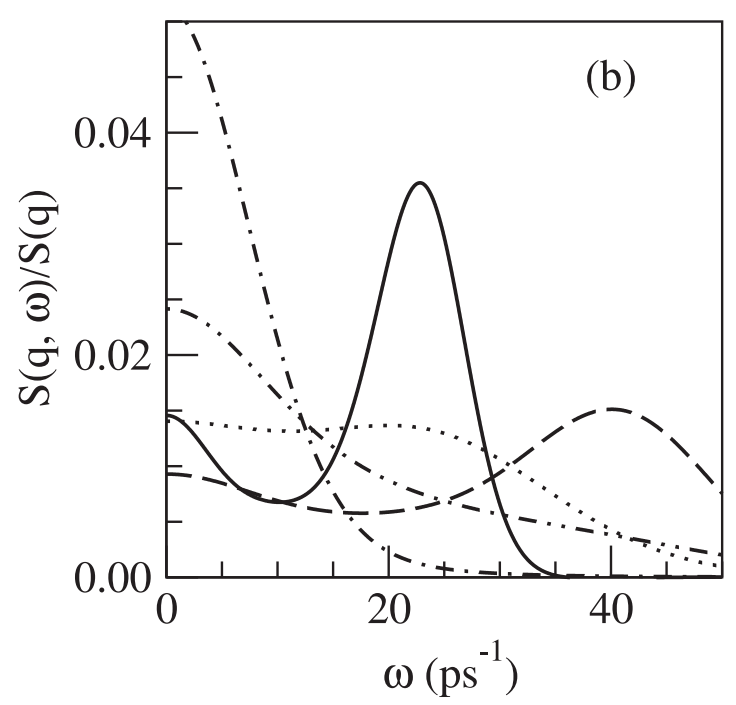

(b)

Figure 8. (a) Intermediate scattering function of $\mathrm{l}-\mathrm{Cu}$ at $T=1423 \mathrm{~K}$. Full line: $0.58 \AA^{-1}$, dashed line: $1.5 \AA^{-1}$, dotted line: $2.22 \AA^{-1}$, dotted-dashed line: $3.1 \AA^{-1}$, and double dotted-dashed line: $4.31 \AA^{-1}(\mathrm{~b})$ Same as above but for the dynamic structure factor.

Figure 11 (a) shows the results for the normalized $J_{\mathrm{T}}(q, t)$ of $\mathrm{l}-\mathrm{Cu}$ at $T=1423 \mathrm{~K}$ at several $q$ values. Notice that for small $q$ 's, the corresponding $J_{\mathrm{T}}(q, t)$ decrease slowly but it becomes faster with increasing $q$ values. The corresponding spectrum $J_{\mathrm{T}}(q, \omega)$ is depicted in figure $11(\mathrm{~b})$ and for some intermediate $q$-range we observe an inelastic peak at nonzero frequency. This peak, which reflects the propagation of shear waves in the liquid, does not appear at the smallest value reached by the simulation $\left(q=0.334 \AA^{-1}\right)$ but it already shows up for $q=0.473 \AA^{-1} \approx 0.16 q_{\mathrm{p}}$, and remains up to $q \approx 2.5 q_{\mathrm{p}}$. The associated peak frequency increases with $q$, takes a maximum value at $q \approx q_{\mathrm{p}}$, and then decreases with increasing $q$ as $J_{\mathrm{T}}(q, \omega)$ evolves towards a gaussian shape. In fact, we recall that a similar behaviour has already been reported for the alkali metals [69] where the inelastic peak appears for $q \geqslant 0.07 q_{\mathrm{p}}$. On the other hand, from the position of the peaks in the $J_{\mathrm{T}}(q, \omega)$ we can derive an associated transverse dispersion relation, $\omega_{\mathrm{T}}(q)$, which is plotted in figure 16. The $\omega_{\mathrm{T}}(q)$ shows, for small $q$-values, a linear behaviour which

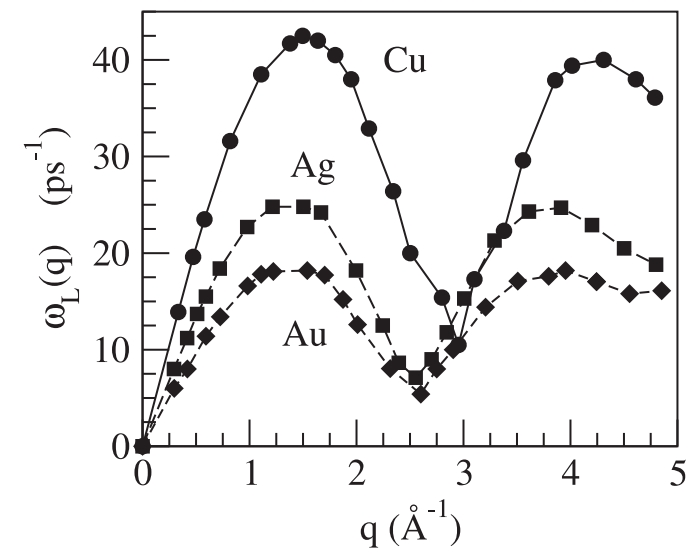

Figure 9. Dispersion relations from the peak positions of the calculated $C_{\mathrm{L}}(q, \omega)=\omega^{2} S(q, \omega)$ for l$\mathrm{Cu}, \mathrm{l}-\mathrm{Ag}$ and $\mathrm{l}-\mathrm{Au}$ at $T=1423,1273$ and $1423 \mathrm{~K}$, respectively.

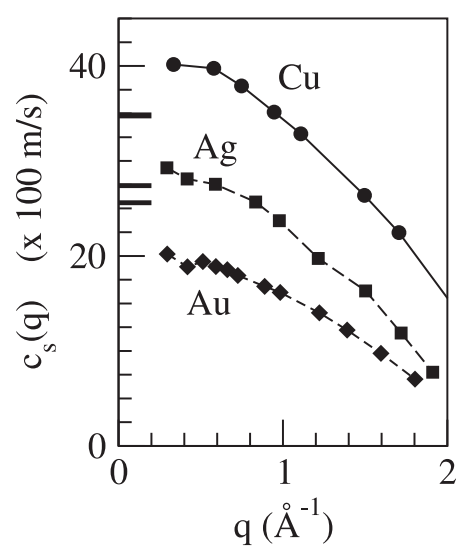

Figure 10. $q$-dependent adiabatic sound velocity for l-Cu, l-Ag and l-Au at $T=1423,1273$ and $1423 \mathrm{~K}$, respectively. The full lines on the $y$-axis stand for the respective hydrodynamic sound velocities. 

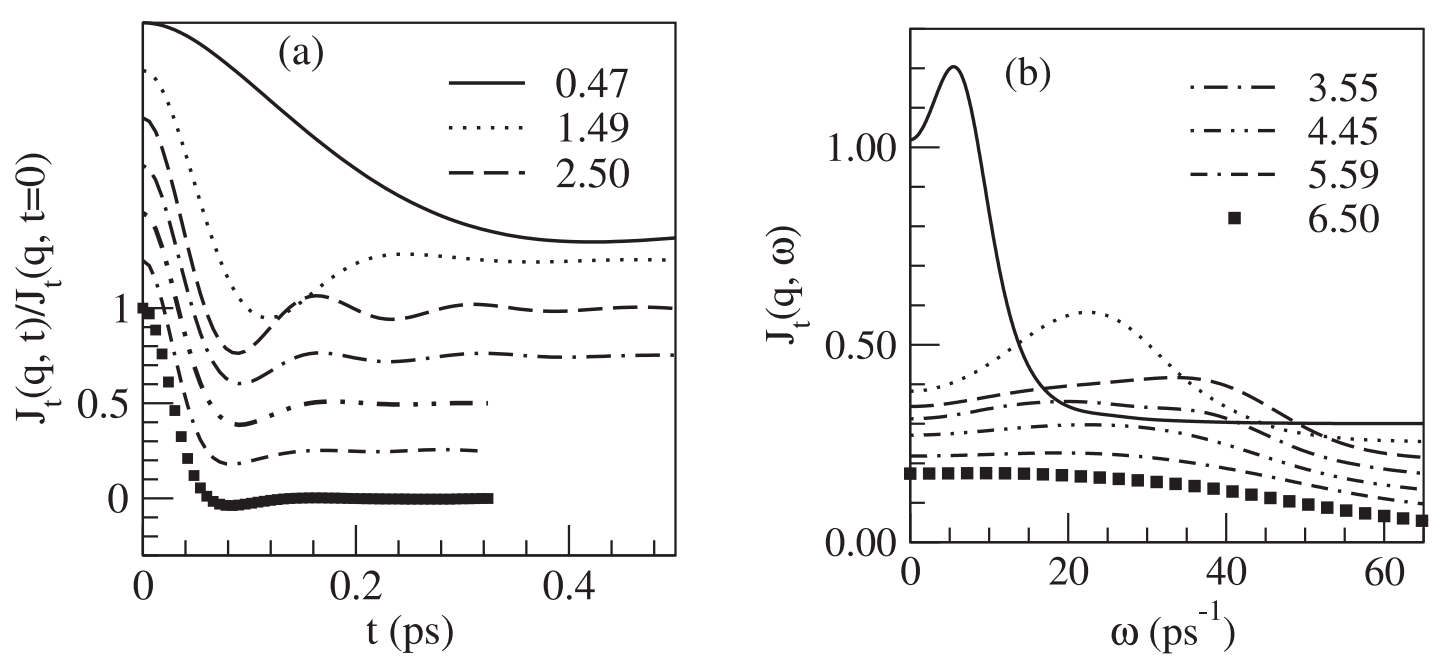

Figure 11. (a) Transverse current correlation function of $\mathrm{l}-\mathrm{Cu}$ at $T=1423 \mathrm{~K}$. (b) Same as above but for the $J_{\mathrm{T}}(q, \omega)$.

can be approximated by $\omega_{\mathrm{T}}(q) \approx c_{\mathrm{t}}\left(q-q_{c}\right)$, where $q_{c}$ is the value at which the $J_{\mathrm{T}}(q, \omega)$ starts showing a maximum and $c_{\mathrm{t}}$ is the velocity of propagation of the shear waves in the liquid metal. In the case of l-Cu at $1473 \mathrm{~K}$, we obtained an estimate $c_{\mathrm{t}} \approx 3000 \pm 200 \mathrm{~m} / \mathrm{s}$.

From the previous $J_{\mathrm{T}}(q, t)$ and using the above mentioned procedure, we evaluated the shear viscosity for $\mathrm{l}-\mathrm{Cu}$. This was performed by calculating the generalized shear viscosity coefficient $\widetilde{\eta}(q)$ for a range $q \leqslant 0.8 \AA^{-1}$ and fitting it to the expression $\widetilde{\eta}(q)=\eta\left(1-\alpha q^{2}\right)$. Herein we estimated $\eta \approx 3.63 \cdot 10^{-3} \mathrm{~kg} / \mathrm{ms}$ (for $T=1423 \mathrm{~K}$ ) and $2.25 \cdot 10^{-3} \mathrm{~kg} / \mathrm{ms}(T=1773 \mathrm{~K}$ ) which compare rather well with the corresponding experimental data of $3.98 \cdot 10^{-3} \mathrm{~kg} / \mathrm{ms}$ and $2.39 \cdot 10^{-3} \mathrm{~kg} / \mathrm{ms}$, respectively [30].

\subsubsection{Liquid Ag}

Figure 12 (a) shows the calculated $F(q, t) / F(q, t=0)$ at several $q$-values for l-Ag at $T=1273 \mathrm{~K}$. Again, for small $q$-values we observe an oscillatory behaviour which is gradually dampened with increasing $q$-values until it finally disappears at $q \approx 0.75 q_{\mathrm{p}}$. The corresponding $S(q, \omega)$ are plotted in figure 12 (b)
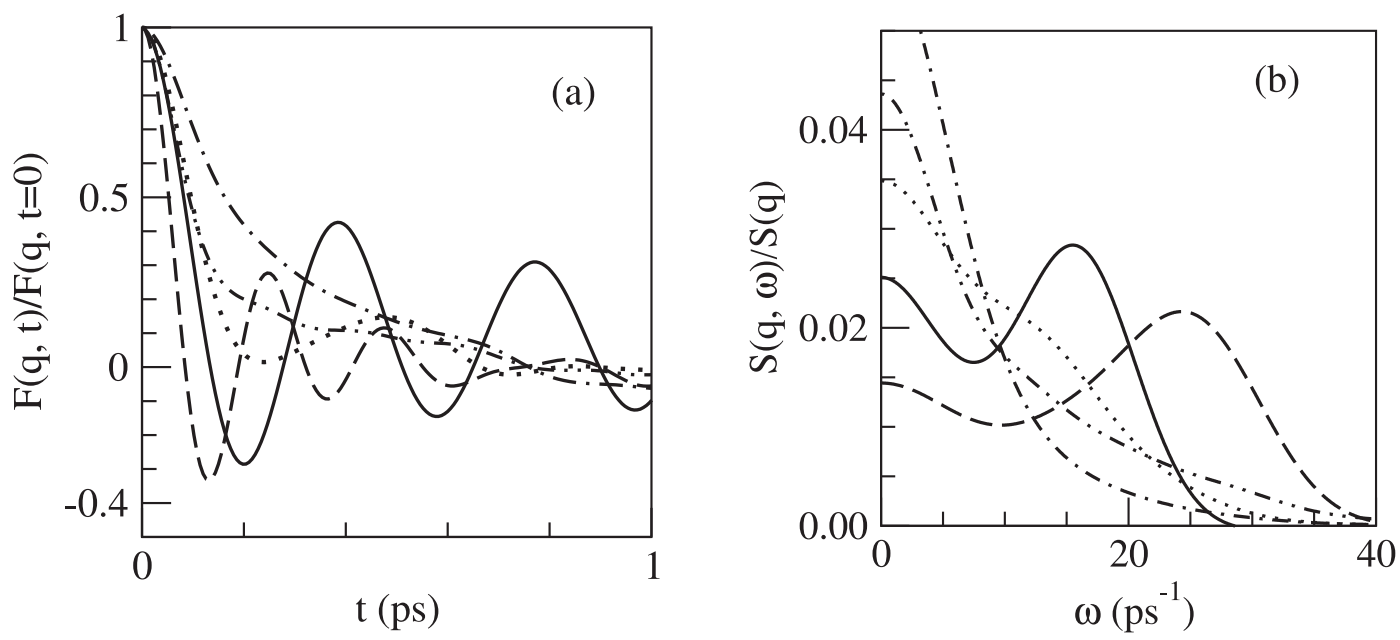

Figure 12. (a) Intermediate scattering function of l-Ag at $T=1273 \mathrm{~K}$. Full line: $0.59 \AA^{-1}$, dashed line: $1.5 \AA^{-1}$, dotted line: $2.1 \AA^{-1}$, dotted-dashed line: $3.0 \AA^{-1}$, and double dotted-dashed line: $4.2 \AA^{-1}$ (b) Same as above but for the dynamic structure factor. 
and we observe side-peaks for a range of small $q$-values, namely up to $q \approx 0.75 q_{\mathrm{p}}$. From the position of the peaks of $J_{\mathrm{L}}(q, \omega)$ we obtain the corresponding dispersion relation, $\omega_{\mathrm{L}}(q)$, plotted for $T=1273 \mathrm{~K}$ in figure 9. Using the smallest $q$-value provided due to the simulations, $q_{\min }=0.294 \AA^{-1}$, we get an estimate $c_{\mathrm{S}}\left(q_{\mathrm{min}}\right) \approx 2930 \mathrm{~m} / \mathrm{s}$, which is somewhat greater than the hydrodynamic values of $c_{\mathrm{s}}=2751$ [27], 2710 [75] or $2797 \mathrm{~m} / \mathrm{s}$ [28], and suggests the existence of a small positive dispersion effect. At a higher temperature, $T=1673 \mathrm{~K}$, our calculation predicts a value of $2720 \mathrm{~m} / \mathrm{s}$, whereas the experimental adiabatic sound velocity is $2663 \mathrm{~m} / \mathrm{s}$ [28]. We are not aware of any inelastic XR or neutron scattering experiments for liquid Ag to compare with.
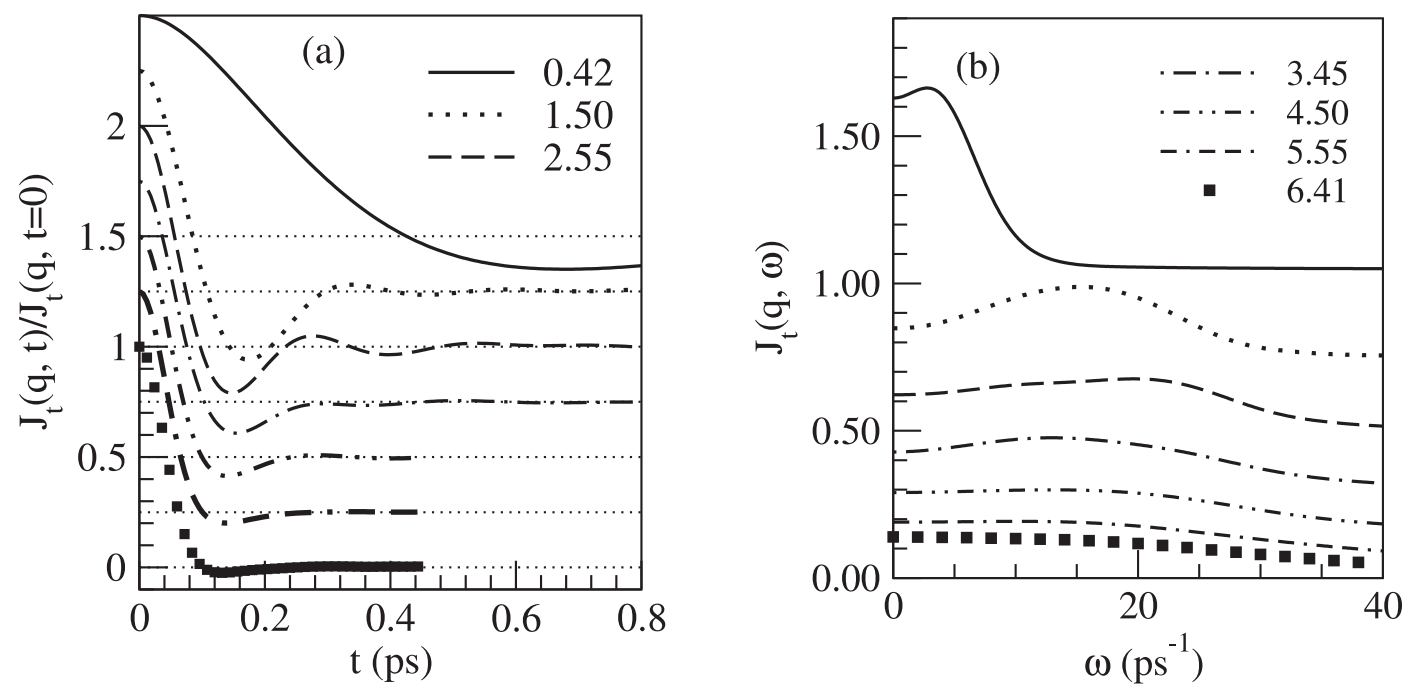

Figure 13. (a) Transverse current correlation function of l-Ag at $T=1273 \mathrm{~K}$. (b) Same as above but for the $J_{\mathrm{T}}(q, \omega)$.

Figure 13 shows the calculated $J_{\mathrm{T}}(q, t)$ and their Fourier Transforms for $\mathrm{l}-\mathrm{Ag}$ at $T=1423 \mathrm{~K}$ and for several $q$ values. The main features for $J_{\mathrm{T}}(q, t)$ and $J_{\mathrm{T}}(q, \omega)$ are similar to those found in l-Cu. The $J_{\mathrm{T}}(q, \omega)$ shows the peaks from which the corresponding dispersion relation $\omega_{\mathrm{T}}(q)$ was calculated and it is depicted in figure 16. Again, a linear fit of the small $q$ values yields an estimate of $c_{\mathrm{t}} \approx 1950 \pm 150 \mathrm{~m} / \mathrm{s}$ for the velocity of the associated shear waves. The calculation of the shear viscosity yields $\eta=3.48 \cdot 10^{-3} \mathrm{~kg} / \mathrm{ms}$ and $2.16 \cdot 10^{-3}$ at $T=1273 \mathrm{~K}$ and $1673 \mathrm{~K}$, which is in good agreement with the respective experimental data $\eta=3.69 \cdot 10^{-3}$ and $2.23 \cdot 10^{-3} \mathrm{~kg} / \mathrm{ms}$ [30].

\subsubsection{Liquid Au}

Figure 14 (a) shows the calculated $F(q, t) / F(q, t=0)$ for several $q$-values. Their main features are similar to those found in l-Cu and l-Ag, namely the existence of oscillations up to $q \approx 0.75 q_{\mathrm{p}}$. Figure 14(b) depicts, for several $q$-values, the corresponding $S(q, \omega)$ which show clear side-peaks up to $q \approx 0.75 q_{\mathrm{p}}$. The dispersion relation of the longitudinal currents is plotted in figure 9 for $T=1423 \mathrm{~K}$. From the smallest $q$-value in the simulations, $q_{\min }=0.296 \AA^{-1}$, we get an estimate $c_{\mathrm{s}}\left(q_{\min }\right) \approx 2030 \mathrm{~m} / \mathrm{s}$, which is clearly below the hydrodynamic adiabatic value of $c_{\mathrm{s}}=2567 \mathrm{~m} / \mathrm{s}$ for $1337 \mathrm{~K}$ [27, 31] and $2513 \mathrm{~m} / \mathrm{s}$ at $1423 \mathrm{~K}$ [28]. This points towards the presence of negative dispersion in the dispersion relation. Although negative dispersion has been indeed found and explained in some systems, such as supercritical fluids [76], as a consequence of an increased ratio between the high-frequency sound velocity and the adiabatic one, driven mainly by a decreased value of the density, the present case of liquid Au near melting certainly does not fit into this category of liquids. Two scenarios could possibly explain the negative dispersion obtained in our calculation. The first one is related to the value of the isothermal speed of sound, $c_{\mathrm{T}}$. Blairs' data [27] are consistent with a value of $\gamma=1.50$ at $1337 \mathrm{~K}$, which yields a value of $c_{\mathrm{T}}=2096 \mathrm{~m} / \mathrm{s}$, so that at $1423 \mathrm{~K}$, the isothermal sound velocity would be somewhat smaller, i.e., quite similar to our result for $c_{\mathrm{s}}\left(q_{\mathrm{min}}\right)$. Singh et al.'s data [28] give, however, a value of $\gamma=1.36$ at $1336 \mathrm{~K}$, and $\gamma=1.40$ at 

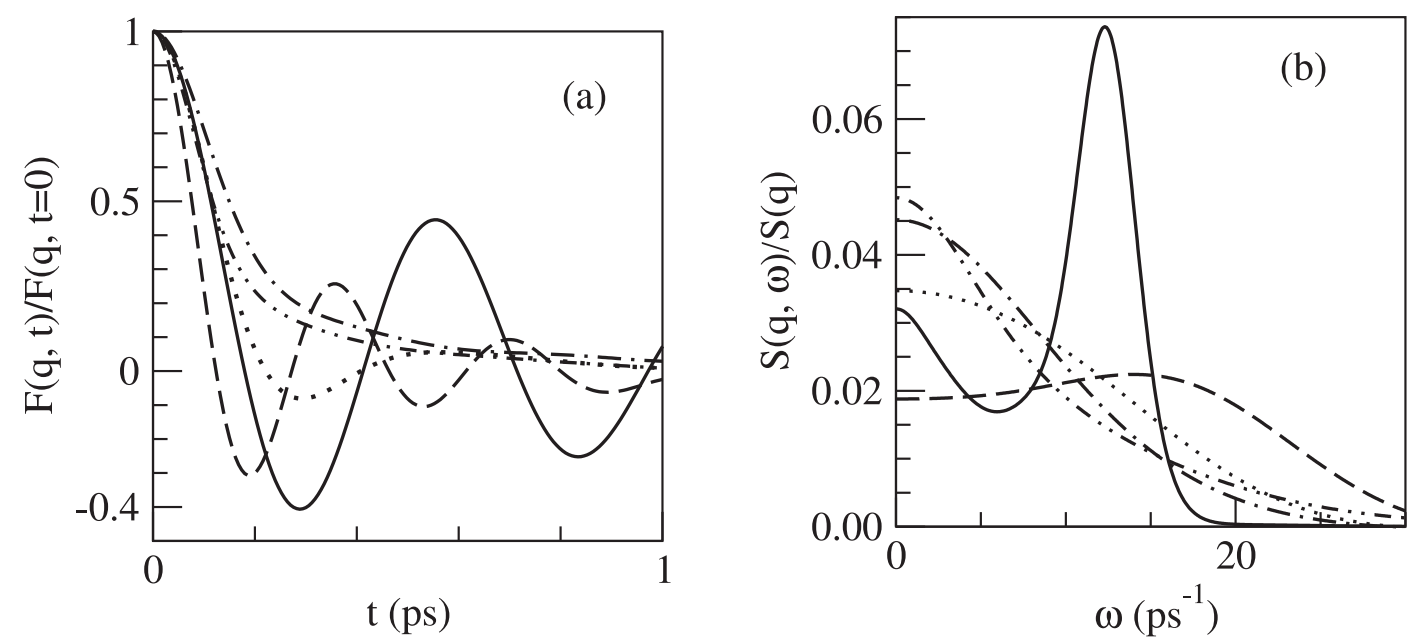

Figure 14. (a) Intermediate scattering function of l-Au at $T=1423 \mathrm{~K}$. Full line: $0.59 \AA^{-1}$, dashed line: $1.39 \AA^{-1}$, dotted line: $2.0 \AA^{-1}$, dot-dashed line: $3.2 \AA^{-1}$ and double dot-dashed line: $4.2 \AA^{-1}$ (b) Same as above but for the dynamic structure factor.

$1423 \mathrm{~K}$, producing an isothermal sound velocity of $2124 \mathrm{~m} / \mathrm{s}$ at $1423 \mathrm{~K}$, which is also similar, although still larger, than our $c_{\mathrm{s}}\left(q_{\mathrm{min}}\right)$ We could, therefore, argue that we are in a wavenumber domain where sound propagation is isothermal in nature. This effect was indeed found in l-Ni [74, 77] and it is connected with the existence of an intermediate isothermal domain standing between hydrodynamic and highfrequency domains [78]. The second scenario is simply a scenario of either inaccuracies in the theoretical method that lead to a simulation adiabatic sound velocity which is too small compared with experiment, or inaccuracies in the experimental data which would report too high a value of the real sound velocity. In either case, the negative dispersion that we find would just be an artifact produced by the wrong value of the hydrodynamic sound velocity. In this respect, it is worth recalling that just one measurement of the speed of sound in liquid Au exists [29]. It is also worth mentioning that very few theoretical calculations of this property can be found in the literature. For instance, the only KS-AIMD simulations of liquid Au [49] did not address the collective dynamics. Concerning CMD, we have only found one reference where a value of $c_{\mathrm{S}}$ is mentioned [79], which was obtained using a glue model interatomic potential, successfully used previously to study several properties of solid Au; the value of $c_{\mathrm{S}}$ was $3700 \mathrm{~m} / \mathrm{s}$ at $1360 \mathrm{~K}$, which is notoriously high as compared to our result and the experimental value.
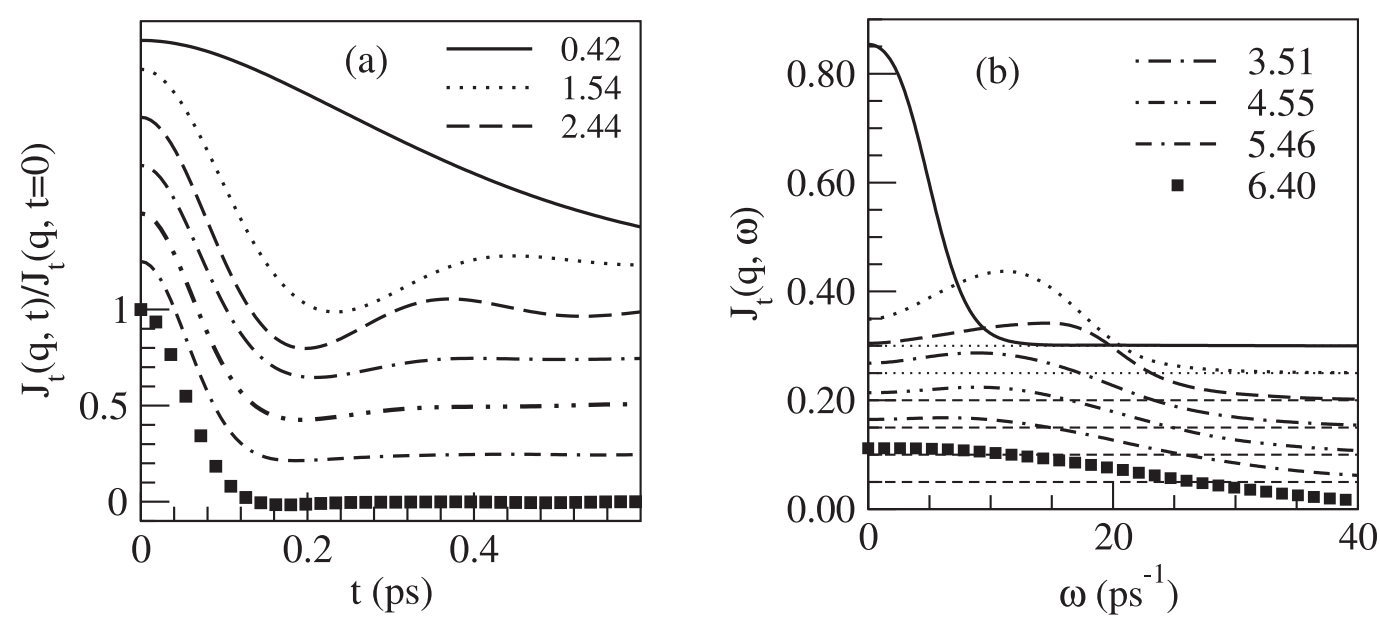

Figure 15. (a) Transverse current correlation function of l-Au at $T=1423 \mathrm{~K}$. (b) Same as (a) but for the $J_{\mathrm{T}}(q, \omega)$. 


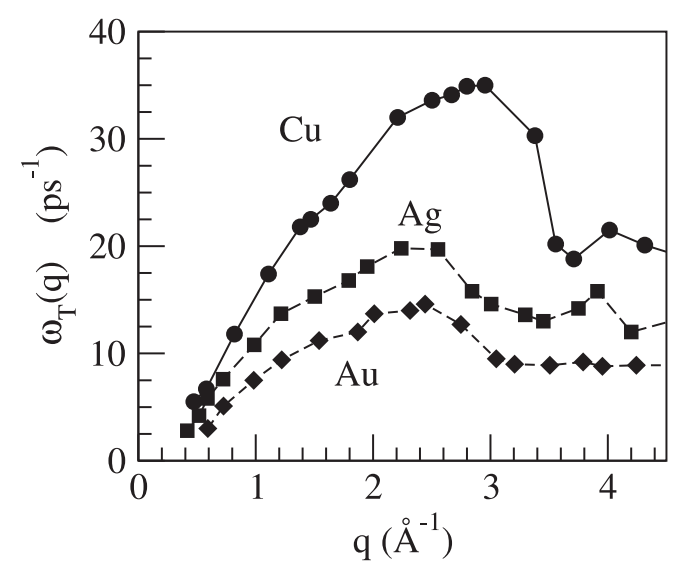

Figure 16. Transverse dispersion relations for $\mathrm{l}-\mathrm{Cu}, \mathrm{l}-\mathrm{Ag}$ and $\mathrm{l}-\mathrm{Au}$ at $\mathrm{T}=1423,1273$ and $1423 \mathrm{~K}$, respectively.

Figure 15 shows the calculated $J_{\mathrm{T}}(q, t)$ and $J_{\mathrm{T}}(q, \omega)$ for l-Au at $T=1423 \mathrm{~K}$ and several $q$ values. The corresponding transverse dispersion relation is plotted in figure 16. Its low $q$ behaviour leads to an estimate $c_{\mathrm{t}} \approx 1380 \pm 150 \mathrm{~m} / \mathrm{s}$ for the velocity of the corresponding shear waves. The calculation of the shear viscosity has yielded values $\approx 4.05 \cdot 10^{-3} \mathrm{~kg} / \mathrm{ms}$ and $3.304 \cdot 10^{-3} \mathrm{~kg} / \mathrm{ms}$ for $T=1423 \mathrm{~K}$ and $1773 \mathrm{~K}$, respectively; these are close to the corresponding experimental data of $4.34 \cdot 10^{-3} \mathrm{~kg} / \mathrm{ms}$ and $3.33 \cdot 10^{-3} \mathrm{~kg} / \mathrm{ms}$ [30].

\section{Conclusion}

We have reported several static and dynamic properties of the liquid noble metals ( $\mathrm{Cu}, \mathrm{Ag}, \mathrm{Au}$ ), each at two thermodynamic states near their respective triple points. This was carried out by using the orbital free ab-initio molecular dynamic simulation method which has already shown its capability for yielding accurate estimates of the same properties for a range of simple metals and alloys [55, 56].

The static structure of the three systems at the two temperatures is globally very well described. Only the low- $q$ part of the structure factor differs from the values obtained from thermodynamic data in l-Cu, where $S(0)$ is underestimated and in l-Au where it is overestimated. The close similarities between the structures of l-Ag and l-Au near melting are indeed reproduced within our model. For both systems, the main peaks in their respective $g(r)$ and $S(q)$ are located at very similar positions. This can be explained by noting that the static structure is mostly determined by the repulsive part of the interionic interaction and density of ions. Figure 1 shows that the repulsive part of the non-Coulombic part of the electron ion interaction for $\mathrm{l}-\mathrm{Ag}$ and $\mathrm{l}-\mathrm{Au}$ are practically coincidental whereas the experimental ionic number densities near melting are $0.0551 \AA^{-3}(\mathrm{l}-\mathrm{Ag})$ and $0.0525 \AA^{-3}(\mathrm{l}-\mathrm{Au})$.

As for the dynamic properties, we begin by noting that the calculated $Z(t)$ show the characteristic shape of high density systems [55, 56, 69] (i.e., the simple liquid metals near melting), which can be explained in terms of the so-called cage effect, namely, a tagged particle is enclosed in a cage formed by its adjacent neighbors. Results have also been reported for the selfdiffusion coefficients, adiabatic sound velocities and shear viscosities. The calculated dynamic structure factors, $S(q, \omega)$, show side-peaks up to $q \approx 0.75 q_{\mathrm{p}}$, which is similar, albeit a bit larger than that of the simple liquid metals [55, 56, 69]. The calculated dispersion relations suggest the existence of some positive dispersion in l-Cu and, to a smaller extent, in l-Ag; in the case of l-Au, some negative dispersion appears to happen, but we could not ellucidate whether it is a real feature or it is due to inaccuracies in the experimental data or in the theoretical model.

We conclude, as far as the agreement with the available experimental data is concerned the present OF-AIMD results for static and dynamic properties are very good. Most importantly, the results also show the capability and reliability of our approach in handling very complicated $d$-electron systems in liq- 
uid phase from the perspective of ab-initio studies. Additional OF-AIMD calculations combined with our model for the pseudopotential are already in progress for several liquid transition metals. Preliminary results are very encouraging and those will be reported in due course.

\section{Acknowledgements}

This work was supported by MICINN in conjunction with the EU FEDER funds (project FIS2011-22957) and by Junta de Castilla y Leon (project VA104A11-2). DJG additionally acknowledges financial support from MECD (project PR2011-0019). GMB is grateful to the Universidad de Valladolid for a fellowship which allowed him to carry out this work. He also gratefully acknowledges the hospitality provided by Professor D. J. González and L. E. González during his stay at the Universidad de Valladolid.

\section{References}

1. Friedel J., The Physics of Metals 1. Electrons, ed. Ziman J.M. Cambridge University Press, 1971.

2. Pettifor D.G., J. Phys. F: Metal Phys., 1977, 7, 613; doi 10.1088/0305-4608/7/4/013

3. Sayers C.M., J. Phys. F: Metal Phys., 1977, 7, 1157; doi 10.1088/0305-4608/7/7/017

4. Wills J.M., Harrison W.A., Phys. Rev. B, 1983, 28, 4363; doi 10.1103/PhysRevB.28.4363

5. Moriarty J.A., Phys. Rev. B, 1982, 26, 1754; doi 10.1103/PhysRevB.26.1754

6. Moriarty J.A., Phys. Rev. B, 1988, 28, 3199; doi 10.1103/PhysRevB.38.3199

7. Moriarty J.A., Phys. Rev. B, 1990, 42, 1609; doi 10.1103/PhysRevB.42.1609

8. Bretonnet J.L., Silbert M., Phys. Chem. Liq., 1992, 24, 169; doi 10.1080/00319109208027266

9. Hausleitner Ch., Kahl G., Hafner J., J. Phys. Condens. Matter, 1991, 3, 1589; doi 10.1088/0953-8984/3/11/017.

10. Aryasetiawan F., Silbert M., Stott M.J., J. Phys. F: Metal Phys., 1986, 16, 1419; doi 10.1088/0305-4608/16/10/011

11. Dagens L., J. Phys. F: Metal Phys., 1986, 16, 1705; doi 10.1088/0305-4608/16/11/011

12. Andersen O.K., Phys. Rev. B, 1975, 12, 3060; doi 10.1103/PhysRevB.12.3060.

13. Soler J.M., Williams A.R., Phys. Rev. B, 1990, 42, 9728; doi 10.1103/PhysRevB.42.9728

14. Yu R., Singh D., Krakauer H., Phys. Rev. B, 1992, 43, 6411; doi 10.1103/PhysRevB.43.6411

15. Daw M.S., Baskes M.I., Phys. Rev. Lett., 1983, 50, 1285; doi $10.1103 /$ PhysRevLett.50.1285

16. Finnis M.W., Sinclair J.E., Phil. Mag. A, 1984, 50, 45; doi 10.1080/01418618408244210

17. Foiles S.M., Phys. Rev. B, 1985, 32, 3409; doi 10.1103/PhysRevB.32.3409

18. Voter A.F., Chen S.P., Mater. Res. Soc. Symp. Proc., 1986, 82, 175; doi 10.1557/PROC-82-175

19. Chen S.P., Srolovig D.J., Voter A.F., J. Mater. Res., 1989, 4, 62; doi 10.1557/JMR.1989.0062

20. Oh D.J., Johnson R.A., J. Mater. Res., 1988, 3, 471; doi 10.1557/JMR.1988.0471

21. Bhuiyan G.M., Silbert M., Stott M.J., Phys. Rev. B, 1996, 53, 636; doi 10.1103/PhysRevB.53.636

22. Gelatt C.D.Jr., Ehrenreich H., Watson R.E., Phys. Rev. B, 1977, 15, 1613; doi 10.1103/PhysRevB.15.1613

23. Do Phuong L., Pasturel A., Nguyen Manh D., J. Phys.: Condens. Matter, 1993, 5, 1901; doi $10.1088 / 0953-8984 / 5 / 13 / 008$

24. Waseda Y., The Structure of Non-Crystalline Materials. McGraw-Hill, New York, 1980.

25. Eder O.J., E. Erdpresser, Kunsch B., Stiller H., Suda M., J. Phys. F: Metal Phys., 1980, 10, 183; doi $10.1088 / 0305-4608 / 10 / 2 / 008$

26. Bellissent M.C., Desre P., Bellissent R., Tourand G., J. Phys., 1976, 37, 1437; doi 10.1051/jphys:0197600370120143700

27. Blairs S., Int. Mater. Rev., 2007, 52, 321; doi $10.1179 / 174328007 X 212490$

28. Singh R.N., Arafin A., George A.K., Physica B, 2007, 387, 344; doi 10.1016/j.physb.2006.04.029

29. Bek R., Steeb S., Phys. Chem. Liq., 1977, 6, 113; doi 10.1080/00319107708084134

30. Shimoji M., Itami T., Atomic Transport in Liquid Metals. Trans Tech Publications, Switzerland, 1986.

31. Iida T., Guthrie R.I.L., The Physical Properties of Liquid Metals. Clarendon, Oxford, 1988.

32. Meyer A., Phys. Rev. B, 2010, 81, 012102; doi 10.1103/PhysRevB.81.012102

33. Hansen J.P., McDonald I.R., Theory of Simple Liquids. Academic Press, London, 1986.

34. Holender J.M., Phys. Rev. B, 1990, 41, 8054; doi 10.1103/PhysRevB.41.8054

35. Bogicevic A., Hansen L.B., Lundqvist B.I., Phys. Rev. E, 1997, 55, 5535; doi 10.1103/PhysRevE.55.5535

36. Alemany M.M.G., Calleja M., Rey C., Gallego L.J., Casas J., González L.E., J. Non-Cryst. Solids, 1999, 250-252, 53; doi 10.1016/S0022-3093(99)00216-1

37. Alemany M.M.G., Gallego L.J., González L.E., González D.J., J. Chem. Phys., 2000, 113, 10410; doi $10.1063 / 1.1322626$ 
38. Alemany M.M.G., Dieguez O., Rey C., Gallego L.J., Phys. Rev. B, 1999, 60, 9208; doi 10.1103/PhysRevB.60.9208

39. Alemany M.M.G., Rey C., Gallego L.J., J. Chem. Phys., 1998, 109, 5175; doi 10.1063/1.477133

40. Han X.J., Chen M., Lu Y.J., Int. J. Thermophys., 2008, 29, 1408; doi 10.1007/s10765-008-0489-7

41. Bhuiyan G.M., Bretonnet J.L., González L.E., Silbert M., J. Phys.: Condens. Matter, 1992, 4, 7651; doi $10.1088 / 0953-8984 / 4 / 38 / 002$

42. Bhuiyan G.M., Bretonnet J.L., Silbert M., J. Non-Cryst. Solids, 1993, 156-158, 145; doi 10.1016/0022-3093(93)90149-R

43. Hohenberg P., Kohn W., Phys. Rev., 1964, 136, B864; doi 10.1103/PhysRev.136.B864

44. Kohn W., Sham L.J., Phys. Rev., 1965, 140, A1133; doi 10.1103/PhysRev.140.A1133

45. Pasquarello A., Laasonen K., Car R., Lee C., Vanderbilt D., Phys. Rev. Lett., 1992, 69, 1982; doi 0.1103/PhysRevLett.69.1982.

46. Mitrohkin Yu., Comp. Mat. Science, 2006, 36, 189; doi 10.1016/j.commatsci.2005.07.002

47. Ganesh P., Widom M., Phys. Rev. B, 2006, 74, 234205; doi 10.1103/PhysRevB.74.134205

48. Fang H.Z., Hui X., Chen G.L., Liu Z.K., Phys. Lett. A, 2008, 372, 5831; doi 10.1016/j.physleta.2008.07.022

49. Pasturel A., Tasci E.S., Sluiter M.H.F., Jakse N., Phys. Rev. B, 2010, 81, 140202; doi 10.1103/PhysRevB.81.140202

50. Vanderbilt D., Phys. Rev. B, 1990, 41, 7892; doi 10.1103/PhysRevB.41.7892

51. Pearson M., Smargiassi E., Madden P.A., J. Phys.: Condens. Matter, 1993, 5, 3221; doi 10.1088/0953-8984/5/19/019.

52. Vichare A., Kanhere D.G., J. Phys.: Condens. Matter, 1998, 10, 3309; doi 10.1088/0953-8984/10/15/008

53. Aguado A., López J.M., Alonso J.A., Stott M.J., J. Chem. Phys., 1999, 111, 6026; doi 10.1063/1.479899.

54. Foley M., Smargiassi E., Madden P.A., J. Phys.: Condens. Matter, 1994, 6, 5231; doi 10.1088/0953-8984/6/28/002

55. González D.J., González L.E., López J.M., Stott M.J., J. Chem. Phys., 2001, 115, 2373; doi 10.1063/1.1389473

56. González D.J., González L.E., López J.M., Stott M.J., Phys. Rev. B, 2002, 65, 184201; doi 10.1103/PhysRevB.65.184201

57. Bhuiyan G.M., González L.E., González D.J., Euro. Phys. J. Web of Conferences, 2011, 15, 01011; doi 10.1051/epjconf/20111501011.

58. Trouiller N., Martins J.L., Phys. Rev. B, 1991, 43, 1993; doi 10.1103/PhysRevB.43.1993

59. Calderín L., González D.J., González L.E., López J.M., J. Chem. Phys., 2008, 129, 194506; doi 10.1063/1.3020304

60. Calderín L., González L.E., González D.J., Phys. Rev. B, 2009, 80, 115403; doi 10.1103/PhysRevB.80.115403

61. Itami T., Munejiri S., Masaki T., Aoki H., Ishil Y., Kamiyama T., Senda Y., Shimojo F., Hoshino K., Phys. Rev. B, 2003, 67, 064201; doi 10.1103/PhysRevB.67.064201

62. Blanco J., González D.J., González L.E., López J.M., Stott M.J., Phys. Rev. E, 2003, 67, 041204; doi 0.1103/PhysRevE.67.041204

63. González D.J., González L.E., López J.M., Stott M.J., Phys. Rev. E, 2004, 69, 031205; doi 10.1103/PhysRevE.69.031205

64. González L.E., González D.J., López J.M., J. Phys.: Condens. Matter, 2001, 13, 7801; doi 10.1088/0953-8984/13/34/321

65. Arai T., Yokoyama I., Waseda Y., J. Non-Cryst. Solids, 1990, 117/118, 96; doi 10.1016/0022-3093(90)90887-R

66. Shimoji M., Liquid Metals. Academic Press, London, 1977.

67. Cusak N.E., The Physics of Structurally Disordered Matter. Adam-Hilger, Bristol, 1987.

68. McGreevy R.L., Baranyai A., Ruff I., Phys. Chem. Liq., 1986, 16, 47; doi 10.1080/00319108608078499

69. Balucani U., Zoppi M., Dynamics of the Liquid State. Clarendon, Oxford, 1994.

70. Casas J., González D.J., González L.E., Alemany M.M.G., Gallego L.J., Phys. Rev. B, 2000, 62, 12095; doi 10.1103/PhysRevB.62.12095

71. González L.E., González D.J., Canales M., Z. Phys. B: Cond. Matter, 1996, 100, 601; doi 10.1007/s002570050167

72. Canales M., González D.J., González L.E., Padró J.A., Phys. Rev. E, 1998, 58, 4747; doi 10.1103/PhysRevE.58.4747

73. Palmer B.J., Phys. Rev. E, 1994, 49, 359; doi 10.1103/PhysRevE.49.359

74. Scopigno T., Ruocco G., Rev. Mod. Phys., 2005, 77, 881; doi 10.1103/RevModPhys.77.881

75. Beyer R.T., Ring E.M., Liquid Metals: Chemistry and Physics, ed. S.Z. Beer. Marcel, New York, 1972.

76. Bryk T., Mryglod I., Scopigno T., Ruocco G., Gorelli F., Santoro M., J. Chem. Phys., 2010, 133, 024502; doi $10.1063 / 1.3442412$

77. Bermejo F.J., Saboungi M.L., Price D.L., Alvarez M., Roessli B., Cabrillo C., Ivanov A., Phys. Rev. Lett., 2000, 85, 106; doi 10.1103/PhysRevLett.85.106

78. Scopigno T., Ruocco G., Phys. Rev. E, 2004, 70, 013201; doi 10.1103/PhysRevE.70.013201

79. Iarlori S., Carnevalli P., Ercolessi F., Tosatti E., Europhys. Lett., 1989, 10, 329; doi 10.1209/0295-5075/10/4/008 


\title{
Дослідження деяких статичних та динамічних властивостей рідких благородних металів методом безорбітальної першопринципної молекулярної динаміки
}

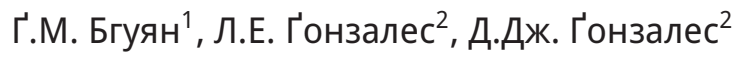 \\ 1 Факультет теоретичної фізики, університет м. Дака, Дака-1000, Бангладеш \\ 2 Факультет теоретичної фізики, університет м. Вальядолід, Вальядолід, Іспанія
}

Оцінено деякі статичні та динамічні властивості рідких $\mathrm{Cu}, \mathrm{Ag}$ i Au при термодинамічних умовах, близьких до їх точок плавлення, за допомогою методу безорбітальної першопринципної молекулярної динаміки. Розрахована статична структура добре узгоджується з наявними даними, отриманими з рентгенівської і нейтронної дифракцій. Щодо динамічних властивостей, то розраховані динамічні структурні фактори вказують на існування колективних збуджень густини поряд з позитивною дисперсією для I-Cu i I-Ag. Отримано деякі коефіцієнти переносу, які прийнятно узгоджуються з наявними експериментальними даними.

Ключові слова: рідкі благородні метали, безорбітальна теорія функціоналу густини, моделювання методом молекулярної динаміки, статична структура, динамічні властивості, коефіцієнти переносу 


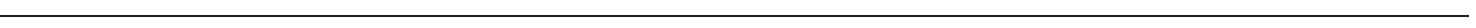

